\title{
Validating dynamicity in resting state fMRI with activation- informed temporal segmentation
}

\author{
Marlena Duda $^{1}$ (1) | Danai Koutra ${ }^{2}$ | Chandra Sripada ${ }^{3}$ (
}

${ }^{1}$ Department of Computational Medicine and Bioinformatics, University of Michigan, Ann Arbor, Michigan, USA

${ }^{2}$ Department of Computer Science and Engineering, University of Michigan, Ann Arbor, Michigan, USA

${ }^{3}$ Department of Psychiatry, University of Michigan, Ann Arbor, Michigan, USA

\section{Correspondence}

Marlena Duda, Department of Computational Medicine and Bioinformatics, University of Michigan, Ann Arbor, Michigan, USA.

Email: marlenad@umich.edu

Chandra Sripada, Department of Psychiatry, University of Michigan, Ann Arbor, Michigan, USA.

Email: sripada@umich.edu

\section{Funding information}

University of Michigan, Grant/Award Number: Precision Health Investigator Award; National Science Foundation Graduate Research Fellowship Program, Grant/Award Number: DGE-1256260

\begin{abstract}
Confirming the presence (or absence) of dynamic functional connectivity ( $\mathrm{dFC}$ ) states during rest is an important open question in the field of cognitive neuroscience. The prevailing dFC framework aims to identify dynamics directly from connectivity estimates with a sliding window approach, however this method suffers from several drawbacks including sensitivity to window size and poor test-retest reliability. We hypothesize that time-varying changes in functional connectivity are mirrored by significant temporal changes in functional activation, and that this coupling can be leveraged to study $\mathrm{dFC}$ without the need for a predefined sliding window. Here, we introduce a data-driven dFC framework, which involves informed segmentation of fMRI time series at candidate FC state transition points estimated from changes in whole-brain functional activation, rather than a fixed-length sliding window. We show our approach reliably identifies true cognitive state change points when applied on block-design working memory task data and outperforms the standard sliding window approach in both accuracy and computational efficiency in this context. When applied to data from four resting state fMRI scanning sessions, our method consistently recovers five reliable FC states, and subject-specific features derived from these states show significant correlation with behavioral phenotypes of interest (cognitive ability, personality). Overall, these results suggest abrupt whole-brain changes in activation can be used as a marker for changes in connectivity states and provides new evidence for the existence of time-varying FC in rest.
\end{abstract}

\section{KEYWORDS}

brain networks, dynamic functional connectivity, fMRI, functional connectivity

\section{1 | INTRODUCTION}

Over the past two decades the study of functional connectivity (FC) has emerged as a preeminent method in cognitive and clinical neuroscience, aiming to characterize the functional network organization of the brain, and to identify objective markers of neuropsychiatric diseases and clinically relevant phenotypes. FC describes the interconnection (often computed as temporal correlation) in activation patterns of spatially distinct regions of the brain, typically measured by blood oxygen level-dependent (BOLD) functional magnetic resonance imaging (fMRI). Originally, the entire field of FC was built on a critical assumption: that patterns of connectivity are static during any given measurement interval in a resting state, that is, the absence of any cognitive task (Biswal, Yetkin, Haughton, \& Hyde, 1995). Static FC has been used to identify global differences in functional network organization of the brain between cognitive task states and resting 
state (Greicius, Krasnow, Reiss, \& Menon, 2003), as well as to characterize differences in FC between healthy controls and subjects with neuro-psychiatric diagnoses, such as schizophrenia (Lynall, 2010) or autism spectrum disorder (Hull et al., 2017).

Recently, however, a number of studies have questioned this assumption, instead advocating the "dynamic" or "time-varying" connectivity view that functional connectivity patterns exhibit substantial moment-to-moment changes over time, specifically within a standard fMRI measurement interval of 5 to $15 \mathrm{~min}$ (Calhoun, Miller, Pearlson, \& Adalı, 2014; Chang \& Glover, 2010; Cohen, 2018; Hutchison et al., 2013; Lurie et al., 2019; Preti, Bolton, \& Van De Ville, 2017). These changing FC patterns are thought to correspond to cognitively meaningful discrete FC network configurations, or connectivity states, that are reproducible both within and between individual subjects. Dynamic states have been documented across different populations, including children (Marusak et al., 2018) and adults (Allen et al., 2014; Cai et al., 2018; Chen, Cai, Ryali, Supekar, \& Menon, 2016; Choe et al., 2017; Liu \& Duyn, 2013; Smith, Zhao, Keilholz, \& Schumacher, 2018), and have been supported with concurrent electroencephalography (EEG) data (Allen, Damaraju, Eichele, Wu, \& Calhoun, 2018; Chang, Liu, Chen, Liu, \& Duyn, 2013; Tagliazucchi, von Wegner, Morzelewski, Brodbeck, \& Laufs, 2012). Furthermore, it has been shown that other characteristics such as the amount of time spent in specific states and the number of transitions between states vary with meaningful individual differences such as age (Cabral et al., 2017; Hutchison \& Morton, 2015; Marusak et al., 2016), sex (Mao, Zheng, Long, Yao, \& Wu, 2017), or disease status (Cordes et al., 2018; Damaraju et al., 2014; Jones et al., 2012; Rashid, Damaraju, Pearlson, \& Calhoun, 2014).

By definition, the presence of dynamic functional connectivity $(\mathrm{dFC})$ in resting state is marked by changes in the connectivity structure of the fMRI time series. The prevailing sliding window framework aims to identify these second-order changes using functional connectivity "snapshots" obtained from time windows of fixed length slid across the entire fMRI time series. The resultant windowed connectomes are then flattened into feature vectors, concatenated across subjects, and clustered into $k$ distinct connectivity states. Importantly, there are two distinct elements of the sliding window paradigm (windowing and connectome estimation) that present several methodological choices that can be mixed-and-matched to create numerous potential sliding window workflows. For example, the windowing step involves the choice of the size and shape of the window (Mokhtari, Akhlaghi, Simpson, Wu, \& Laurienti, 2019; Shakil, Billings, Keilholz, \& Lee, 2018; Shakil, Keilholz, \& Lee, 2015; Shakil, Lee, \& Keilholz, 2016), the optimal choice of which still constitutes an active area of research. There are also several choices of connectivity estimation, including Pearson correlation (Allen et al., 2014), Spearman correlation (Savva, Mitsis, \& Matsopoulos, 2019), instantaneous shared trajectory (Faghiri et al., 2020), and instantaneous phase synchrony (Pedersen, Omidvarnia, Zalesky, \& Jackson, 2018). Each of these methods presents its own benefits, but Pearson correlation is generally the most commonly used connectivity estimator in sliding window paradigms. The sliding window approach represents an important advance in the study of time-varying brain connectivity, but it nonetheless suffers from several important limitations.

First, the sliding window method relies heavily on the somewhat arbitrary choice of window size, and results can differ substantially across various window widths (Hindriks et al., 2016; Shakil et al., 2016). A second problem is that simulations suggest that sliding window methods can introduce artifactual connectivity variation even under conditions when such variation is known to be absent (Laumann et al., 2017; Lindquist, Xu, Nebel, \& Caffo, 2014). Third, perhaps due to one or more of the preceding issues, the sliding window method has been found to have poor test-retest reliability (Choe et al., 2017). Fourth, the overlapping nature of the sliding windows precludes definitive segmentation of the fMRI time series into states, making interpretation of the state dynamics difficult. Finally, the sliding window approach requires constructing a sizable number of overlapping windowed connectivity matrices: with 400 timepoints and a 30 second window, 370 distinct connectivity matrices are required (at a step $=1 \mathrm{TR}=1 \mathrm{~s}$ ). This poses serious scalability issues for relatively long or more temporally granular fMRI datasets.

Some alternatives to sliding window approaches have been proposed in recent years; however, these too have certain drawbacks and limitations. The dynamic conditional correlation (DCC) model is a multivariate volatility model that estimates the changing covariance structure at each timepoint in the fMRI time series (Choe et al., 2017; Lindquist et al., 2014). While the DCC model allows for a parametric approach to estimating framewise FC with robust statistical inference, it increases the number of connectivity matrices to consider in the final clustering step compared to the sliding window method, further hindering its scalability. Furthermore, the formulation of the DCC model has been shown to give biased results in high dimensional data (Hafner \& Reznikova, 2012), which poses an issue for application in fMRI data with a large number of ROIs and time points. Two other recently proposed moment-to-moment methods, multiplication of temporal derivatives (Shine et al., 2015) and edge co-fluctuations (Esfahlani et al., 2020), have similar formulations and are both aimed at uncovering the degree of functional coupling for all ROI pairs at each timepoint. Similarly to DCC, these methods result in a higher dimensional output than that of the sliding window, and the instantaneous estimates of connectivity at each timepoint are highly susceptible to noise. Hidden Markov models (HMMs), which seek to decompose a time series into a sequence of discrete "hidden" states, are another increasingly popular approach for estimating connectivity dynamics (Baker et al., 2014; Quinn et al., 2018; Vidaurre, Smith, \& Woolrich, 2017; Zhang et al., 2020). However, HMMs rely on several strong assumptions including a predefined number of $k$ hidden states that transition between one another in a Markovian fashion (state transitions depend solely on the state at the previous time point). Moreover, HMMs trained at the group level assume a single governing state-to-state transition structure across all subjects, which may be too strict and miss important individual variability.

Our focus here is on a hybrid approach that bridges windowed and instantaneous methods by leveraging moment-to-moment changes in activation to inform tailored time series segmentation at 
candidate FC state change points, which reduces both the dimensionality and noisiness that affects many other moment-to-moment dFC methods. It is well known from the task-based fMRI literature that task-driven changes in activation patterns co-occur with changes in connectivity patterns (Davison et al., 2015; Gonzalez-Castillo et al., 2015; Shine \& Poldrack, 2018; Spielberg, Miller, Heller, \& Banich, 2015; Sripada et al., 2014; Telesford et al., 2016). This coupling of activation and connectivity changes suggests the possibility that changes in the activation structure of the fMRI time series, which are easily derived, can serve as a reasonably reliable marker for changes in the connectivity structure, which are more difficult to obtain in an unbiased way. Though connectivity changes may not always be accompanied by activation changes, as long as there is significant correspondence, we can leverage the latter (straightforwardly identified) to find the former (less so) without the need for sliding windows.

In this work we leverage the coupling between activation and connectivity to present the activation-informed segmentation approach, a data-driven dFC framework centered around informed segmentation of $\mathrm{fMRI}$ time series at candidate FC state change points. Moment-to-moment changes in functional activations have previously been utilized in the literature to investigate dynamic functional connectivity (Shine et al., 2015), but have yet to be used to localize connectivity state changepoints for dynamic time series segmentation. Our approach detects significant instantaneous changes in functional activation patterns and generates data-driven segments of stable connectivity throughout the fMRI time series. For clarity, we will use the term "segments" when referring to our method and "windows" when referring to the sliding window approach. Separating the time series into discrete time segments rather than a set of highly overlapped sliding windows significantly improves the computational efficiency of dFC analysis and enhances interpretability of results by enabling precise identification of state transition junctures-something the sliding window method cannot provide. We suggest that these FC-tailored segments provide a useful alternative to standard sliding windows in dFC analyses and show that our approach significantly outperforms the sliding window paradigm in recovering known FC state transitions in a block-design task. Furthermore, we propose a framework for the comparison of connectomes derived from segments of variable length, as well as a graph embedding step for summarizing connectomes into low-dimensional representations that we show are better suited for downstream clustering and machine learning tasks than current approaches.

\section{2 | METHODS}

\section{1 | Data description}

\subsection{1 | HCP data}

In this work, we utilize the Human Connectome Project (HCP) S1200 Young Adult dataset made publicly available through the Washington University and the University of Minnesota HCP consortium (http:// humanconnectome.org). It is one of the richest collections of neuroimaging data to date, consisting of structural and functional MRI, behavioral assessments, and genotypes for 1200 healthy subjects ages 2235. A full description of the acquisition protocol can be found in (Van Essen et al., 2013). In short, all HCP fMRI data were acquired on a modified Siemens Skyra $3 \mathrm{~T}$ scanner using multiband gradient-echo $\mathrm{EPI}\left(\mathrm{TR}=720 \mathrm{~ms}, \mathrm{TE}=33 \mathrm{~ms}\right.$, flip angle $=52^{\circ}$, multiband acceleration factor $=8,2 \mathrm{~mm}$ isotropic voxels, FOV $=208 \times 180 \mathrm{~mm}$, 72 slices, alternating RL/LR phase encode direction). Participants completed four total resting state fMRI scanning sessions (two sessions collected on each of two different days). Each resultant resting state fMRI time series consisted of 1200 volumes sampled every $0.72 \mathrm{~s}$, for a total acquisition time of $14 \mathrm{~min}$ and $24 \mathrm{~s}$. During the resting state sessions participants were instructed to keep their eyes open and fixated on a cross hair on the screen, while remaining as still as possible. For clarity, we will refer to resting state data from the first collection day as sessions $1 \mathrm{~A}(\mathrm{RL})$ and $1 \mathrm{~B}(\mathrm{LR})$, and similarly sessions $2 \mathrm{~A}$ and $2 \mathrm{~B}$ for those collected on the second day.

Though our main objective is to assess FC dynamics during rest, we also leverage the repeating task/rest block structure of the working memory (WM) task data available in $\mathrm{HCP}$ as a natural ground truth to test the performance of our method in identifying the known transitions between the task and rest conditions. The HCP WM task consists of four repeating task/rest blocks, where each block is structured as follows: $27.5 \mathrm{~s}$ Task 1 (0-back), $27.5 \mathrm{~s}$ Task 2 (2-back), $15 \mathrm{~s}$ rest. Using the same acquisition details outlined above, each WM task fMRI time series consisted of 405 volumes sampled every $0.72 \mathrm{~s}$, for a total acquisition time of $4 \mathrm{~min}$ and $52 \mathrm{~s}$. Two sessions of WM task fMRI were acquired back-to-back, alternating between RL and LR phase encoding directions. We will refer to these as WM session 1 (RL) and WM session 2 (LR).

\subsection{2 | Data preprocessing}

Processed volumetric data from the HCP minimal preprocessing pipeline including ICA-FIX denoising were used. Full details of these steps can be found in (Glasser et al., 2013; Salimi-Khorshidi et al., 2014). Briefly, BOLD fMRI data were gradient-nonlinearity distortion corrected, rigidly realigned to adjust for motion, fieldmap corrected, aligned to the structural images, and then registered to $\mathrm{MNI}$ space with the nonlinear warping calculated from the structural images. Then FIX was applied on the data to identify and remove motion and other artifacts in the timeseries. These files were used as a baseline for further processing and analysis (e.g., MNINonLinear/ Results/rfMRI_REST1_RL/rfMRI_REST1_RL_hp2000_clean.nii.gz from released $\mathrm{HCP}$ data).

Images were smoothed with a $6 \mathrm{~mm}$ FWHM Gaussian kernel, and then resampled to $3 \mathrm{~mm}$ isotropic resolution. This step as well as the use of the volumetric data, rather than the surface data, were done to allow comparability with other large datasets in ongoing and planned analyses that are not amenable to surface-based processing. The smoothed images then went through a number of resting state 
processing steps, including motion artifact removal steps comparable to the type B (i.e., recommended) stream of (Siegel et al., 2017). Further details on motion artifact removal can be found in (Sripada et al., 2019). Lastly, we calculated spatially averaged time series for each of the 268 ROls from the parcellation given in (Finn et al., 2015).

For our analysis, we first considered the set of 966 subjects listed in (Sripada et al., 2019) that met the following criteria: structural T1 and $\mathrm{T} 2$ data, four complete resting state $\mathrm{fMRI}$ sessions, and $<10 \%$ of resting state frames censored due to excessive motion (framewise displacement of $0.5 \mathrm{~mm}$ ). From this set 922 subjects also had two complete WM task $\mathrm{fMRI}$ sessions, defining our final subset of subjects.

\section{2 | The activation-informed segmentation framework}

Here, we propose a novel framework for identifying dynamic changes in functional connectivity in fMRI time series, termed the activationinformed segmentation method. This method leverages the coupling between changes in connectivity structure and changes in wholebrain activation patterns to produce an intuitive, interpretable, and computationally efficient alternative to the sliding window approach. Our framework consists of three main steps: tailored segmentation of all $\mathrm{fMRI}$ time series, summarization of the functional connectivity within each discovered segment, and finally segregation and characterization of a final set of connectivity states (Figure 1). These steps are detailed in Sections 2.2.1-2.2.3 below.

\subsection{1 | Activation-informed time series segmentation}

The dynamic FC paradigm suggests the presence of significant instantaneous changes in connectivity structure at transition points between two distinct functional states. Using this logic, we sought to identify potential connectivity state transition points within fMRI data and utilize them to perform informed segmentation of the time series as a means for assessing FC dynamics. Based on the phenomenon established in task-based literature (Davison et al., 2015; GonzalezCastillo et al., 2015; Shine \& Poldrack, 2018; Spielberg et al., 2015; Sripada et al., 2014; Telesford et al., 2016), we hypothesize that changes in the activation structure of the fMRI time series, which are easily derived, can serve as a reasonably reliable marker for changes in the connectivity structure, which are more difficult to obtain in an unbiased way. To estimate the changes in functional connectivity from one time point $t$ to the next, we observe changes in functional activation from one time point to the next by calculating the temporal derivative $(d t)$ of each of $n$ ROI activation time series $(A)$ of length $T$ using first-order differencing similar to that in the multiplication of temporal derivatives (MTD) method (Shine et al., 2015):

$$
d t_{i}(t)=A_{i}(t)-A_{i}(t-1)
$$

At this point, our method importantly diverges from the MTD method: while the MTD uses these ROI-wise temporal derivatives to define the connectivity between each pair of ROls and ultimately generate an $n \times n$ connectome estimate at each time point, our method instead summarizes the regional temporal derivatives to provide a univariate estimate of moment-to-moment changes in activation on the whole brain scale. At this point in our pipeline, the resulting $n$ temporal derivative series of length $T-1$ are summarized by taking the $L_{2}$-norm, that is, the root sum of squares, at each time step $t$, resulting in a single vector of length $T-1$, which we have termed the Global Temporal Derivative (GTD) series:

$$
\operatorname{GTD}(t)=\left\|d t_{1: n}(t)\right\|_{2}=\sqrt{\sum_{i=1}^{n} d_{i}(t)^{2}}
$$

The GTD provides a univariate summarization of instantaneous changes in global brain activation throughout an fMRI time series, therefore peaks in the GTD series correspond to instances of significant moment-to-moment alterations in functional activity. In this way, the GTD is akin to the derivative of the global signal. Growing research suggests the global signal is not noise and carries meaningful

\section{Activation-Informed Segmentation}

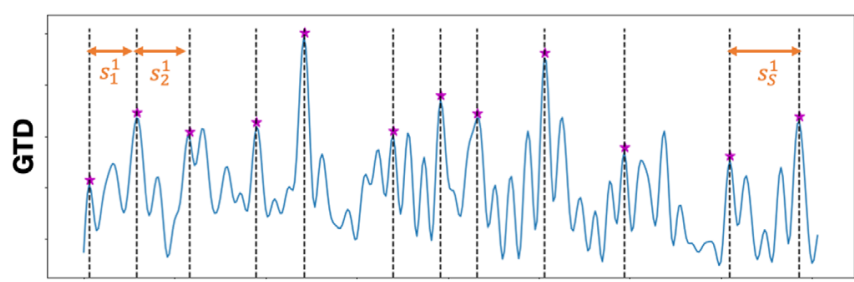

Time
FC Summarization

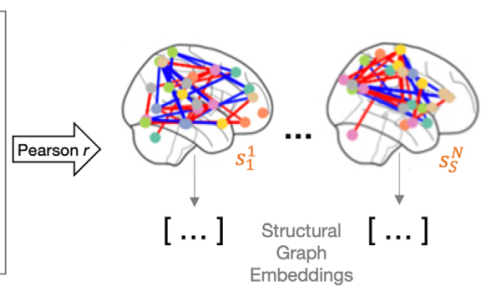

State Characterization

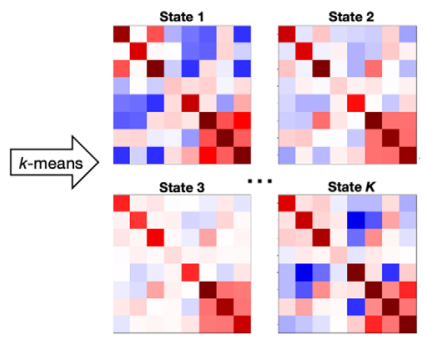

FIGURE 1 Depiction of our activation-informed segmentation pipeline. Briefly, peaks in the GTD series define the boundaries of our tailored, nonoverlapping stable-FC segments $s_{1}$ to $s_{2}$ (note $S$ can vary between subjects) for all subjects 1 to $N$. Next, functional connectivity is summarized using structural graph embeddings for each segment in the set of all segments $\left\{\left\{s_{1}^{1}, s_{2}^{1}, \ldots\right\}, \ldots,\left\{s_{1}^{N}, s_{2}^{N}, \ldots\right\}\right\}$. Finally, $k$-means is applied to segregate all segments into a set of $k$ connectivity states 
information about mental states (Wong, Olafsson, Tal, \& Liu, 2013). Here, we build on this work to suggest that global signal shifts mark changes in dynamic mental states. We seek to automatedly identify these change points as candidate FC state transitions for the subsequent time series segmentation step. We begin by applying exponentially weighted moving average smoothing (window size $=15 \mathrm{TR}$, $\alpha=\frac{1}{(\text { window size }+1)}$ ) to the GTD series to reduce noisy peaks. We then perform moving average peak detection (window size $=20$ TR for Rest, 10 TR for WM task) on the smoothed GTD series, identifying points in the time series that are $>=2.5$ standard deviations above the moving average. To avoid identification of multiple points that surpass this threshold but actually correspond to a single true peak, we collapsed points in close proximity to one another to the local maximum (within $10 \mathrm{TR}$, corresponding to $7 \mathrm{~s}$ or the approximate time-to-peak of the hemodynamic response function [Friston, 2003]). Furthermore, as these change points define our tailored segments for downstream calculation of functional connectivity, we set a minimum inter-peak distance of 25 TR to ensure sufficiently large segments for calculating Pearson correlation (Schönbrodt \& Perugini, 2013; Thirion et al., 2007; Turner, Paul, Miller, \& Barbey, 2018) (note: we reduce this to 15 TR for the case of WM task data to accommodate the shorter resting state segments we intend to capture). This final set of change points define the boundaries of the tailored time segments, within which we compute FC and between which we investigate potential dynamic FC shifts.

\subsection{2 | Functional connectivity estimation}

For each tailored segment $s$, we compute the functional connectivity matrix $C^{(s)}$, where the $i, j^{\text {th }}$ entry is the Pearson correlation of the activation time series of ROls $i$ and $j$ within the time segment, $A_{i}(s)$ and $A_{j}(\mathrm{~s})$ :

$$
\mathrm{C}_{\mathrm{ij}}^{(\mathrm{s})}=\frac{\operatorname{cov}\left(\mathrm{A}_{\mathrm{i}}(\mathrm{s}), \mathrm{A}_{\mathrm{j}}(\mathrm{s})\right)}{\sigma_{\mathrm{A}_{\mathrm{i}}(\mathrm{s})} \sigma_{\mathrm{A}_{\mathrm{j}}(\mathrm{s})}}
$$

We then apply the Fisher transformation followed by z-scoring on each FC matrix $C^{(s)}$, to allow for better comparisons between connectivity matrices of segments of differing lengths. Connectivity matrices derived from shorter segments have, on average, higher correlation values than those from longer segments, resulting in a skewed sample distribution. Applying the Fisher transformation enforces an approximately normal distribution of the connectivity values within each segment (Fisher, 1915), and the z-score then translates these connectivity values in terms of their standard deviations from the mean. While these connectome transformations are common practice in the field of FC, they are especially important when attempting to compare connectomes from segments of variable lengths, which is illustrated in Figure 2.

Thresholding is another common pre-processing step in functional connectivity analysis, as it preserves only the high-fidelity connections within connectomes and effectively filters out noise. Though the Fisher transformation with z-scoring helps to align the sample distributions of connectivity values between longer and shorter segments, we still observed the effects of segment length when thresholding on z-scores alone-connectomes from shorter segments were denser (i.e., had more edges preserved) after thresholding than connectomes from longer segments. This segment-length discrepancy in connectome density with $z$-score thresholding had significant downstream effects in our pipeline, as we found the resultant FC state clusters were highly correlated with segment length. To avoid these segment length effects, we fix the density of all connectomes by thresholding to the top- $K$ connections (or edges) in each connectome. Recent work has suggested that such rank-based schemes are optimal for reliability and reproducibility in FC analyses (Bridgeford et al., 2020). Here, we set top- $K=10,000$, which (a)

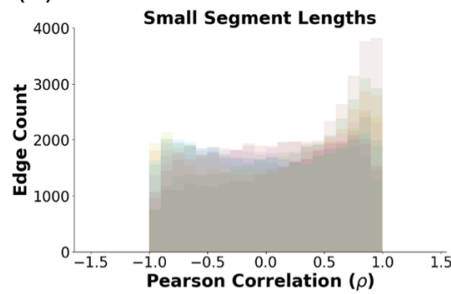

(c)

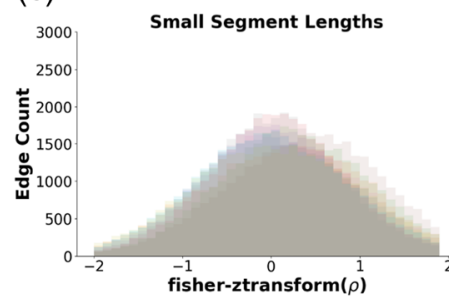

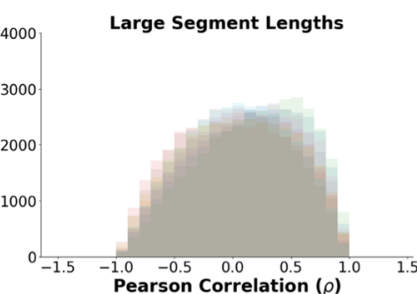

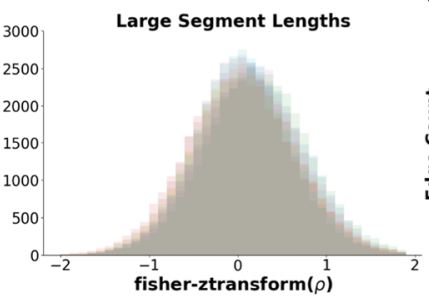

(b)
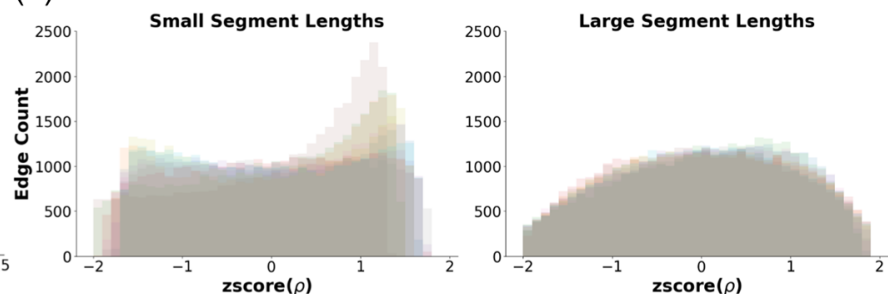

(d)

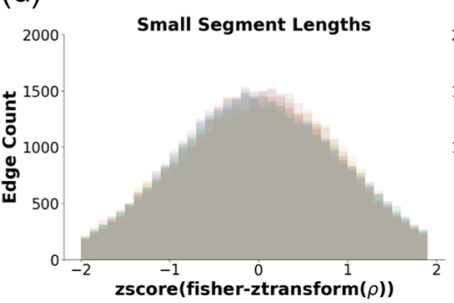

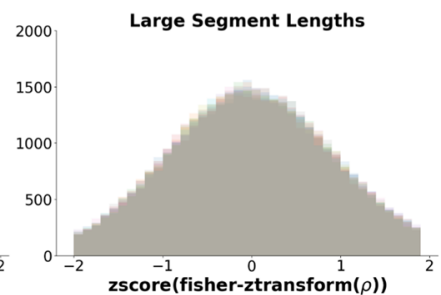

FIGURE 2 Effects of (a) no transformation, (b) z-score transformation, (c) Fisher transformation, and (d) z-scored (Fisher) transformation on the distribution of Pearson correlation-based connectivity values in short ( $<25 T R)$ and long ( $>35 T R)$ segments 
preserves the strongest (i.e., highest magnitude) $27.95 \%$ edges, thereby providing sufficient noise reduction.

\subsection{3 | State clustering}

The final step of our dFC framework involves using $k$-means clustering to separate all thresholded connectomes into a discrete set of $k$ connectivity states. This state clustering occurs on the aggregated set of $m$ connectomes, where $m$ is the total number of time segments across all subjects in a single fMRI scanning session (Table 1). In traditional dFC streams, this approach involves performing $k$-means clustering on the flattened upper triangular of all $m$ connectomes, however we found poor performance with this method, likely due to the high dimensionality of the flattened connectomes $(>35,000)$ (Table S1). We address this issue of high dimensionality by generating low-dimensional latent representations of each thresholded connectivity matrix that sufficiently summarize the connectivity patterns within the time segment. Specifically, we utilize state-of-the-art graph embedding methods, which are commonly used in the field of data mining to generate low-dimensional representations of graphs (i.e., networks) (Rossi et al., 2020). Connectomes are graphs by definition, consisting of a set of nodes (ROIs) connected by edges ( $z$-scored correlations), so graph mining methods naturally extend to the connectome space. To generate our graph embeddings, we first apply
GraphWave (Donnat, Zitnik, Hallac, \& Leskovec, 2018) on the top-Kthresholded connectomes to produce a set of $d$-dimensional node embeddings for each of the $n$ ROIs per connectome. GraphWave learns structural node embeddings, which individually capture the structural role of each node (ROI) within its local network neighborhood and in aggregate provide insights into the topological organization of the connectome graph. We then utilize principal components analysis (PCA) to summarize the set of $n d$-dimensional node embeddings, concatenated into one long node embedding vector of length $n^{*} d$, into a single graph embedding vector by extracting the top 100 principal components. Aggregating these connectome graph embeddings across all time segments from all subjects results in a feature matrix of size $m \times 100$.

We performed $k$-means clustering on the resultant group-level feature matrix, varying the number of clusters $k$ in the range of 2-10. To determine the optimal number of clusters we utilized the elbow criterion of the cluster validity index, computed as the ratio of withincluster distance to between-cluster distance (Allen et al., 2014). We mapped corresponding clusters across the session replicates to a single overall state based on shortest Euclidean distances between the cluster centroid connectomes. Reproducibility of FC state clusters was tested across scanning sessions (two sessions for WM task, four sessions for resting state). Test-retest reliability was calculated across scanning sessions between centroid connectomes of corresponding states using the image intra-class correlation (I2C2) (Shou et al., 2013).

TABLE 1 Symbols and abbreviations

\begin{tabular}{|c|c|c|}
\hline Symbol & Meaning & Value \\
\hline FC & Functional connectivity & - \\
\hline $\mathrm{dFC}$ & Dynamic functional connectivity & - \\
\hline WM & Working memory & - \\
\hline ROI & Region of interest & - \\
\hline GTD & Global temporal derivative & - \\
\hline N & Number of subjects & $N=922$ \\
\hline $\mathrm{n}$ & Number of ROls & $n=268$ \\
\hline$d t$ & Temporal derivative & - \\
\hline$A_{i}$ & Activation time series of $\mathrm{ROI} i$ & - \\
\hline$T$ & Length of time series & $T_{\mathrm{WM}}=405, T_{\mathrm{REST}}=1200$ \\
\hline$t$ & Time point $t$ & - \\
\hline$s, S$ & $\begin{array}{l}\text { Time segment } s \text { and total number of } \\
\text { segments } S \text {, respectively }\end{array}$ & - \\
\hline$C^{(s)}$ & $\begin{array}{l}\text { Functional connectivity matrix for time } \\
\text { segment } s\end{array}$ & - \\
\hline$A_{i}(s)$ & $\begin{array}{l}\text { Activation time series of ROI } i \text { in time } \\
\text { segment } s\end{array}$ & - \\
\hline K & $\begin{array}{l}\text { Number of edges retained in top- } K \\
\text { thresholding }\end{array}$ & $K=10,000$ \\
\hline k & Number of clusters in $k$-means clustering & $k=(2-10)$ \\
\hline$m$ & $\begin{array}{l}\text { Total number of time segments/ } \\
\text { connectomes across all subjects in a } \\
\text { single fMRI scanning session }\end{array}$ & $\begin{array}{l}m_{W M 1}=8740, m_{W M 2}=9052 \\
m_{\text {REST1A }}=16,104, m_{\text {REST } 1 B}=16,015 \\
m_{\text {REST } 2 A}=15,420, m_{\text {REST } 2 B}=16,062\end{array}$ \\
\hline$d$ & Dimensionality of graph embedding & $d=100$ \\
\hline
\end{tabular}


I2C2 is the generalization of the intra-class correlation (ICC) coefficient to high-dimensional multivariate data, such as images (or in our case, connectomes). As a brief description, let $X_{i}(c)$ be the true, unknown connectome for state $i$ and $W_{i j}(c)$ be the estimated connectome for state $i$ during session $j$ at connectome edge $c$. The classical measurement error model for the connectome images across replication studies can then be written as

$$
\mathrm{W}_{\mathrm{ij}}(\mathrm{c})=\mathrm{X}_{\mathrm{i}}(\mathrm{c})+\mathrm{U}_{\mathrm{ij}}(\mathrm{c})
$$

where connectomes are represented as $C \times 1$ vectors; $W_{i j}=\left\{W_{i j}(c)\right.$ : $c=1, \ldots, C\}$ are the observed connectomes; $X_{i}=\left\{X_{i}(c): c=1, \ldots, C\right\}$ are the true connectomes, and $U_{i j}=\left\{U_{i j}(c): c=1, \ldots, C\right\}$ are the measurement error of the connectomes. In this framework, $i=1, \ldots, l$, where $I=$ total states $=5$, and $j=1, \ldots, J_{i}$, where $J_{i}=$ total sessions $=4$. Connected to the classical measurement error model above and analogous to the standard ICC formulation, the I2C2 is defined as:

$$
12 \mathrm{C} 2=\frac{\operatorname{trace}\left(\mathrm{K}_{\mathrm{X}}\right)}{\operatorname{trace}\left(\mathrm{K}_{\mathrm{W}}\right)}=\frac{\operatorname{trace}\left(\mathrm{K}_{\mathrm{W}}\right)-\operatorname{trace}\left(\mathrm{K}_{\mathrm{U}}\right)}{\operatorname{trace}\left(\mathrm{K}_{\mathrm{W}}\right)}=1-\frac{\operatorname{trace}\left(\mathrm{K}_{\mathrm{U}}\right)}{\operatorname{trace}\left(\mathrm{K}_{\mathrm{W}}\right)}
$$

where $K_{U}=\operatorname{cov}\left(U_{i j}, U_{i j}\right), K_{X}=\operatorname{cov}\left(X_{i}, X_{i}\right)$, and $K_{W}=\operatorname{cov}\left(W_{i j}, W_{i j}\right)$, and both $K_{U}$ and $K_{X}$ cannot be estimated directly since $U_{i j}$ and $X_{i}$ are unobserved. Therefore, the $12 \mathrm{C} 2$ is computed using the following method of moments estimators:

$$
\begin{aligned}
& \operatorname{trace}\left(K_{U}\right)=\frac{1}{\sum_{i=1}^{l}\left(J_{i}-1\right)} \sum_{i=1}^{l} \sum_{j=1}^{J_{i}} \sum_{c=1}^{c}\left\{W_{i j}(c)-\bar{W}_{i .}(c)\right\}^{2} \\
& \operatorname{trace}\left(K_{W}\right)=\frac{1}{\sum_{i=1}^{l}\left(J_{i}-1\right)} \sum_{i=1}^{I} \sum_{j=1}^{J_{i}} \sum_{c=1}^{C}\left\{W_{i j}(c)-\bar{W}_{. .}(c)\right\}^{2}
\end{aligned}
$$

where $\bar{W}_{i .}(c)=\frac{\sum_{j=1}^{J_{i}} W_{i j}(c)}{J_{i}}$ is the average connectome for state $i$ over all sessions $j$, and $\bar{W}_{. .}(c)=\frac{\sum_{i, j, c} W_{i j}(c)}{I J}$ is the average connectome across all states and sessions. Utilizing these estimators, $12 \mathrm{C} 2$ metrics were computed in $\mathrm{R}$ using the package provided by the authors in Neuroconductor (https://rdrr.io/github/neuroconductor/I2C2/man/I2C2. $\mathrm{html})$. We further characterize the resultant connectivity states with standard dFC features including average dwell time and state-to-state transition probabilities, and go on to correlate these dFC features with neurophenotypes of interest.

\subsection{Evaluation against ground truth}

As described in Section 2.1.1, the WM task consists of four repeating task/rest blocks, where each block is structured as follows: $27.5 \mathrm{~s}$ Task 1 (0-back), $27.5 \mathrm{~s}$ Task 2 (2-back), $15 \mathrm{~s}$ rest. This repeating task/rest block structure of the WM Task data serves as a natural ground truth for validation of our framework: if activation changes can truly be used as markers for connectivity changes, then we should be able to show that the discovered activation-informed change points align well with true onsets of WM task conditions. In fMRI data, signals are expected to be observed shortly after the stimulus, rather than directly aligned to the stimulus onset, due to lag in the hemodynamic response. Furthermore, the nature of block-design tasks results in sustained task-related activation changes rather than instantaneous spikes and subjects may require an additional 1-2 $\mathrm{s}$ after the condition onset to fully enter the task state and experience the full effects of the task-induced activation response. Based on this, we defined a state change response window of 12 TR $(8.6 \mathrm{~s})$ to account for the hemodynamic response time of 10 TR (7.2 s) as well as an additional buffer of 2 TR (1.4 s) for subjects to fully enter the task condition state. All peaks identified in the GTD series were labeled as either true positives or false positives based on whether they fell within the state change response window following a known task condition transition or not. Based on these labels, we calculate the overall precision and recall of our activation-informed change point detection, as well as the recall for transitions into each of the three task conditions (Task 1, Task 2, and Rest).

\subsection{Comparison to sliding window}

While the sliding window framework has been widely used to estimate dynamic FC states in resting fMRI where ground truth state changes cannot be known, it has not, to the best of our knowledge, been validated against a block-design task structure where the ground truth state changes are in fact known. To enable a direct comparison with the performance of our activation-informed segmentation method we applied the sliding window framework to the WM task data using the Group ICA of fMRI toolbox (GIFT) (https:// trendscenter.org/software/gift/; Center for Translational Research in Neuroimaging and Data Science, Atlanta, Georgia) implementation, following the parameterization detailed in (Allen et al., 2014) as closely as possible. Specifically, we first performed group-level spatial independent component analysis (gICA) (Calhoun, Adali, Pearlson, \& Pekar, 2001) to extract 50 independent components (ICs). IC time series then underwent a standard postprocessing procedure to remove low-frequency trends associated with scanner drift, motion related variance and any other nonspecific "spikes" or possible noise artifacts. Next, we utilized the dFNC function in the GIFT toolbox to perform the sliding window analysis. As in (Allen et al., 2014), we use a tapered window created by convolving a rectangle (window size $=44 \mathrm{~s} / 61 \mathrm{TR}$ ) with a Gaussian ( $\sigma=3 \mathrm{TR}$ ) and sliding in steps of $1 \mathrm{TR}$, resulting in 344 total windows per WM fMRI session, and a total of 317,168 windows across all 922 subjects for each WM Sessions 1 and 2. Finally, the upper triangular of the windowed connectomes were used as feature vectors of length $(50 \times(49)) / 2=1225$, and $k$ means clustering was applied to separate all windows into a set of $k$ states. We utilized the "estimate_clusters" option in the GIFT toolbox to identify the optimal value of $k$ from the range of 2-10. Further details regarding the implementation of the GIFT toolbox steps can be found in the software manual (https://trendscenter.org/trends/ software/gift/docs/v4.0b_gica_manual.pdf). To evaluate the accuracy of the resultant sliding window state clustering and compare against 
that of our proposed method, we implemented the common design choice of setting the ground truth label (i.e., "task" or "rest") for each window as the label assigned to the time point at the center of the window, in this case timepoint 31.

\section{3 | RESULTS}

\section{1 | The GTD method accurately identified known transitions during a working memory task}

Results of GTD-based peak discovery in WM task data are shown in Figure 3. The distribution of the discovered GTD peaks across all subjects showed a concentration of peaks immediately after a new condition onset (Figure 3B). In fMRI data, signals are expected to be observed shortly after the stimulus, rather than directly aligned to the stimulus onset, due to lag in the hemodynamic response. Using the true positive and false positive labels detailed earlier in Section 2.4, we found an average precision of 0.72 and average recall of 0.66 of all discovered change points against ground truth state transitions (Table 2). We found that Task 1 and Rest state onsets were more readily identifiable by our method than Task 2 onsets (Recall 0.67 , 0.75 , and 0.57 , respectively), indicating that transitions from task state to rest state and vice-versa elicit more significant changes in momentto-moment activations than transitions from an easier 0-back WM task (Task 1) to a more difficult 2-back WM task (Task 2).

We found the optimal number of clusters $k=3$ for both WM Session 1 and WM Session 2. Figure 3A illustrates the alignment of our segments, colored by their respective clusters, to the ground truth WM task conditions. Overall, we found good segregation between task and rest conditions, with improved accuracy in later block repetitions. As observed with the change point detection, the separation between Tasks 1 and 2 conditions is more difficult, owing both to the similarity in connectivity between the two working memory task conditions and to the lack of change point detection at Task 2 onset points resulting in segments that span the time frame of both Tasks 1 and 2 . Homogeneity and normalized mutual information (NMI) metrics of our discovered clusters compared to the known ground truth are reported in Table 2. As our temporal segments may not directly align to the ground truth task blocks we derived ground truth labels for each discovered segment based on the corresponding task condition throughout the majority of the segment. (a)

(b)
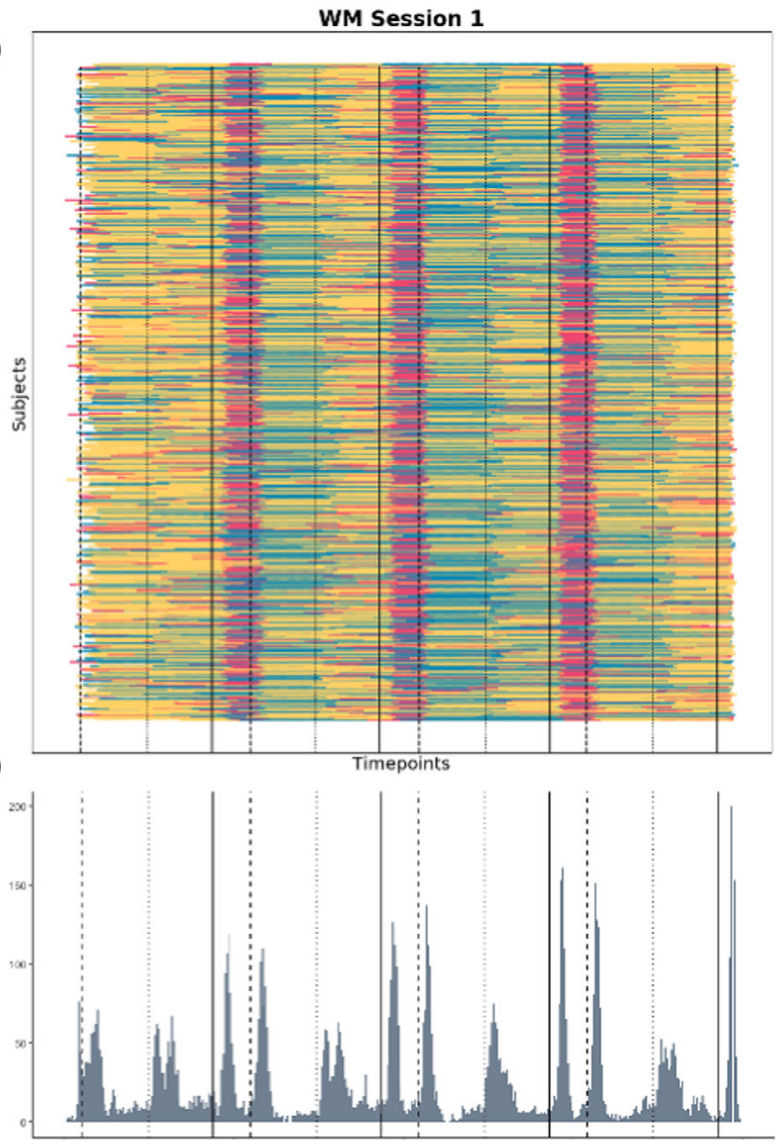

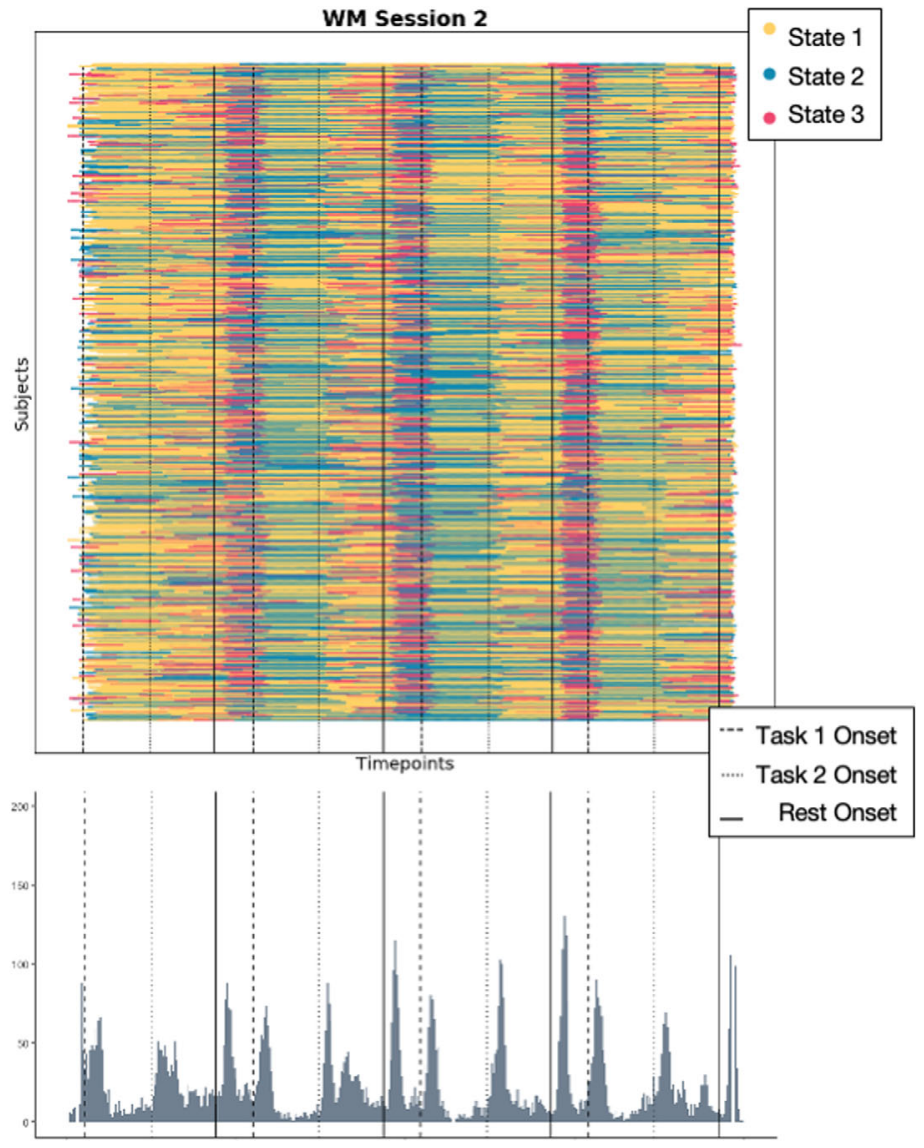

FIGURE 3 Results of the activation-informed segmentation for all subjects in structured WM task data. (A) Temporal alignment of our discovered segments colored by their corresponding state labels given by $k$-means clustering shows good alignment to known ground truth conditions (onsets marked by vertical lines: dashed for Task 1 onset, dotted for Task 2 onset, solid for Rest onset). (B) Histogram of discovered GTD peak locations show strong alignment to known condition onsets 
TABLE 2 Performance of the activation-informed segmentation method and the standard sliding window method in recovering ground truth dynamic state changes in WM task data

\begin{tabular}{|c|c|c|c|c|c|c|c|}
\hline Pipeline step & Metric & \multicolumn{3}{|c|}{ Activation-informed segmentation } & \multicolumn{3}{|l|}{ Sliding window } \\
\hline \multirow[t]{4}{*}{ Change point discovery } & Overall Precision & 0.74 & 0.70 & 0.72 & - & - & - \\
\hline & Overall Recall & 0.67 & 0.64 & 0.66 & - & - & - \\
\hline & Task 1 Recall & 0.72 & 0.62 & 0.67 & - & - & - \\
\hline & Task 2 Recall & 0.54 & 0.59 & 0.57 & - & - & - \\
\hline \multirow[t]{3}{*}{ Clustering } & Optimal $k$ & 3 & 3 & 3 & 5 & 5 & 5 \\
\hline & Homogeneity & 0.327 & 0.233 & 0.280 & 0.037 & 0.037 & 0.037 \\
\hline & NMI & 0.231 & 0.159 & 0.195 & 0.018 & 0.018 & 0.018 \\
\hline
\end{tabular}

Note: The change point discovery step is unique to our framework and unable to be reported for the sliding window method.

\subsection{In the working memory task, activation- informed segmentation performance was superior to sliding window}

We report the results of the GIFT toolbox sliding window pipeline for $k=5$ states, which was estimated as the optimal $k$ using the automated cluster estimation available in the GIFT toolbox (Table 2). Though the sliding window approach does capture some repeating task versus rest signal (Figure 4), we found the GIFT sliding window approach had significantly decreased performance in segregating between known task and rest condition windows compared to our activation-informed segmentation approach (homogeneity $=0.037$ vs. 0.280 , respectively). Based on these results, we can conclude that our method more effectively and efficiently summarized the FC in each time segment, resulting in a $99.8 \%$ reduction in size of the final feature set passed to $k$-means compared to that of the sliding window approach $(8740 \times 100$ vs. $3,17,168 \times 1225$ in WM Session 1). Furthermore, our method proved to be much more computationally efficient than the sliding window approach, completing in $<2$ hours for all subjects in a single WM session while the GIFT toolbox required $>24$ hours to complete the requisite ICA and dFNC steps for the same data. Considering together the accuracy, data reduction and the runtime, we found our activation-informed segmentation method to outperform the traditional sliding window paradigm in recovering dynamics in the context of a block-design ground truth.

\section{3 | The activation-informed segmentation method identified five connectivity states during rest}

We applied our activation-informed segmentation pipeline separately on four sessions of resting state fMRI data. Using the elbow criterion of the cluster validity index, we consistently found the optimal number of clusters $k=5$ across the four sessions (Figure 5). Though our state clusters were derived using the graph embedding vectors as described above, we characterized the connectivity of each discovered cluster using the more interpretable top- $K$ thresholded connectomes derived upstream in our pipeline for all segments in each cluster. We mapped corresponding clusters across the four session replicates to a single overall "dynamic state" based on shortest Euclidean distances between the cluster centroid connectomes and found that each centroid was mapped only to one overall state by this criterion, indicating each state did indeed exhibit a unique connectivity signature.

\subsection{Connectivity states during rest exhibit excellent Test-Retest reliability}

To assess the stability of these clusters we use the I2C2 metric, which was developed to assess the reliability of MRI images for a set of subjects across several image acquisition sessions. The $12 \mathrm{C} 2$ metric is a high-dimensional multivariate generalization of the intra-class correlation coefficient for use on images and other multi-dimensional data, such as connectomes (Shou et al., 2013). A brief description of I2C2 and its application in our case can be found in Section 2.2.3 above. We found very high replicability of our states across the four sessions $($ I2C2 $=0.96)$, suggesting that the dynamic states recovered by our method are indeed persistent across subjects and time, and may also be cognitively meaningful.

\section{5 | Activation peaks observed during rest closely resemble peaks found when transitioning in and out of cognitively demanding task states}

We found that the magnitude of the GTD peaks that correspond to our discovered FC change points and define our dynamic states in rest are on the same order and mirror the distribution of the peaks found in the WM task setting (Kullback-Leibler divergence $=0.030$; Figure 6 ). This indicates that the changes in functional brain activity between dynamic states in rest are as strong as those observed when transitioning in and out of a cognitively demanding task state. 

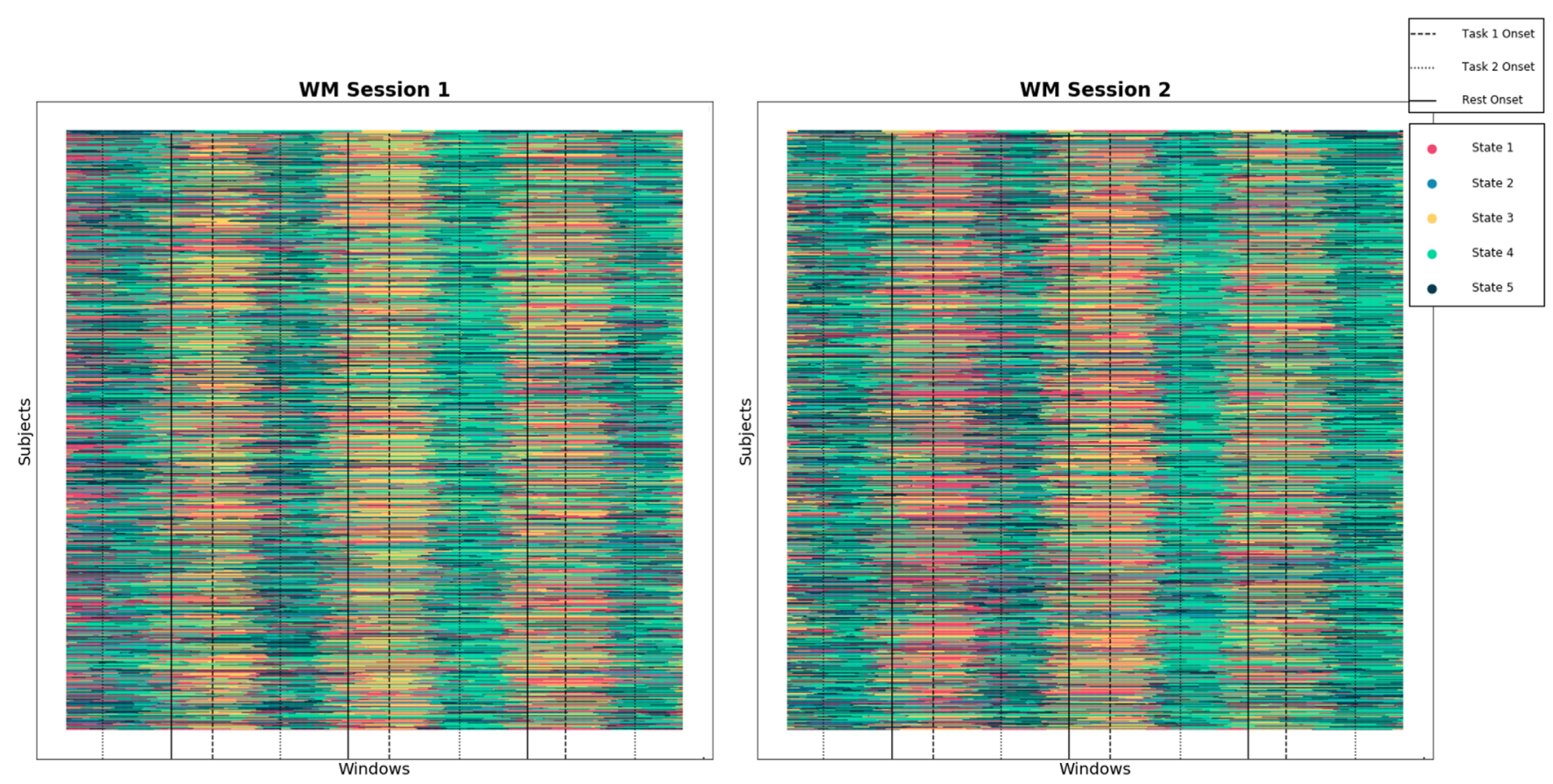

FIGURE 4 Results of GIFT toolbox-based sliding window framework for all subjects in structured WM task data. Task condition onsets marked by vertical lines: dashed for Task 1 onset, dotted for Task 2 onset, solid for Rest onset

\subsection{Connectivity states involve brain-wide connectivity patterns and prominently involve prefrontal/sensory-motor coupling}

We further characterized the overall connectivity signature of each resultant dynamic state by averaging the corresponding cluster centroids across the four sessions. This signature connectome for each of the five overall dynamic states is presented in Figure 7. Overall, we observed states that reflect shifting connectivity across network modules, rather than within network modules, consistent with prior work (Betzel, Fukushima, He, Zuo, \& Sporns, 2016; Zalesky, Fornito, Cocchi, Gollo, \& Breakspear, 2014). In particular, we observed changing patterns of brain integration and segregation, prominently involving the frontoparietal network and the default mode network (Zalesky et al., 2014). States 1, 3 , and 5 all involve sensory/motor anti-correlation with the frontoparietal network and default mode network. State 1 encompassed all sensory and motor networks, while State 3 had greater visual network specificity and State 5 had greater motor specificity. State 2 was characterized by anticorrelation between frontoparietal and medial frontal network, without sensory/motor involvement. State 4 exhibited none of the above motifs-just the within network connectivity that was common to all of the states. Importantly, the five states we observed are highly similar to the states identified in this same HCP dataset using the classic sliding window paradigm as reported in Nomi et al., (2017).

\section{7 | Resting connectivity states exhibit complex patterns of transitioning}

In addition to summarizing each dynamic state by its unique connectivity patterns, we also extracted common dFC features including state-to-state transition probabilities, average dwell times per state, and number of occurrences of each state across the four resting sessions. We extracted these dFC features on a per-subject basis and then averaged them to capture the general patterns for all five dynamic states at the group level. The average state-to-state transition matrix, average dwell times, and average number of occurrences per state across all subjects are depicted in Figure 8. Overall, we found the highest probabilities of transitioning into State 4 from any of the other states. Interestingly, State 4 also exhibits the shortest dwell time of all five states, averaging a duration of $29.8 \pm 2.5 \mathrm{~s}$, as well as the highest average number of occurrences. This coupled with the lower overall connectivity observed in State 4 suggests that this may represent a "buffer" state between the other dynamic states.

\section{8 | Resting connectivity states are correlated with behavioral phenotypes including cognition, personality, and psychopathology}

We performed a regression analysis to assess the combined relationship between subject-specific dFC feature vectors, averaged across the four resting state sessions, and several neuro-relevant phenotypes. Specifically, we consider 10 cognitive metrics: a general factor of intelligence (G; generated from a bifactor model as described in [Sripada, Angstadt, Rutherford, Taxali, \& Shedden, 2020]), processing speed (generated from factor modeling of three NIH Toolbox tasks as described in (Sripada et al., 2019)), the five facets of personality given by the Revised NEO Personality Inventory (openness to experience, conscientiousness, extraversion, agreeableness, and neuroticism), and the three dimensions of psychopathology given by the Adult Self Report Scale (Internalizing, Attention Problems, Externalizing). We also included the 

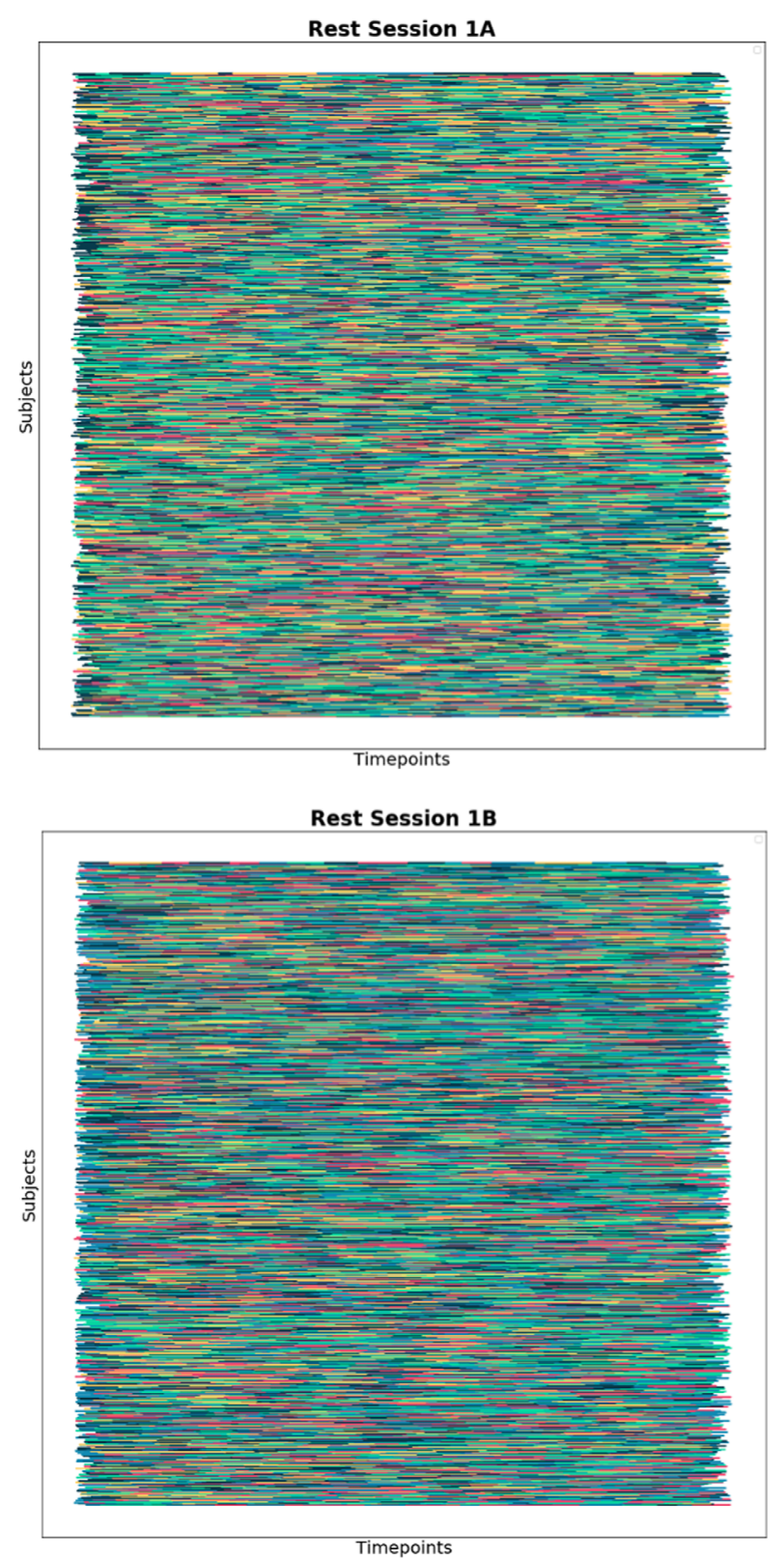
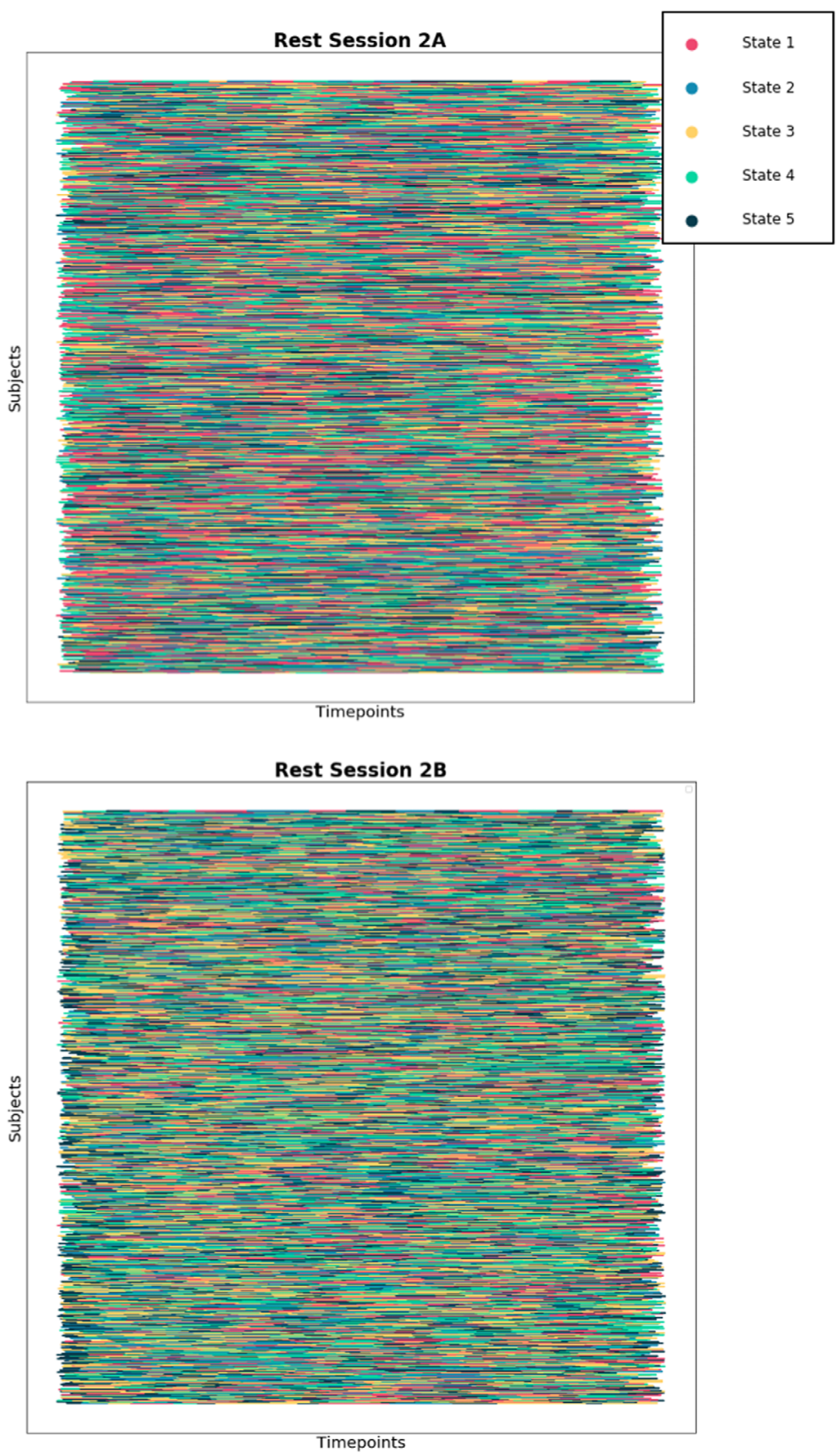

FIGURE 5 Temporal alignment of activation-informed segments and their corresponding state labels given by $k$-means in all four resting state fMRI sessions

covariates of age and gender. All features (besides the binary gender marker) were $z$-scored prior to the regression analysis, so the resultant model $\beta$ values could be interpreted similarly to correlation values. At a Bonferroni-corrected $\alpha=0.005$ significance threshold, we found significant relationships between our $\mathrm{dFC}$ features and four phenotypes (G, externalizing behavior, agreeableness and conscientiousness). Significant regression results are reported in Table 3.

\section{9 | Resting connectivity states are unrelated to head motion}

Head motion is a serious confound in studies of functional connectivity (Power, Barnes, Snyder, Schlaggar, \& Petersen, 2012; Power,
Schlaggar, \& Petersen, 2015; Satterthwaite et al., 2012; van Dijk, Sabuncu, \& Buckner, 2012). Moreover, it has recently been argued that head motion may in fact generate the time varying connectivity observed with sliding window methods (Laumann et al., 2017). We thus sought to determine whether the connectivity states we detected at rest with the GTD method were related to head motion. We found no significant correlation between the mean framewise displacement time series and the GTD series in all four resting state sessions $(r=-0.0027 ; 95 \% \mathrm{Cl}=[-0.006,0.0007])$. We report all time-lagged cross-correlations for \pm 10 TR in each of the four resting state sessions in Table S2. This lack of correlation between framewise displacement and the GTD series suggests that there is no significant contribution of head motion to our discovered change points, and thereby our final dynamic states in rest. Taken together, these results strongly support 
the general existence of dynamicity in resting state and the reliability of the states discovered by our activation-informed framework.

\section{4 | DISCUSSION}

In this study, we introduce a new data-driven approach for assessing dynamic functional connectivity through informed time series

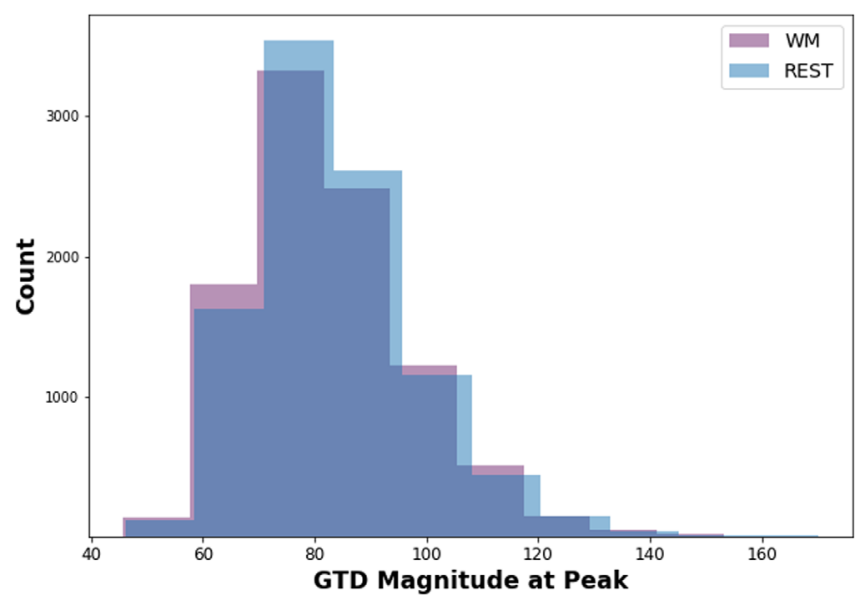

FIGURE 6 Histograms of GTD magnitudes at discovered peaks for 9700 change points in WM Session 1 and a size-matched random sample of change points in Rest Session 1A show similar distributions (Kullback-Leibler divergence $=0.030$ ) segmentation. Our method, termed the activation-informed segmentation method, aims to derive FC states without the limitations of a predefined time scale for the dynamics or highly overlapped sliding windows. This framework is built upon the theory that changes in functional connectivity are mirrored by changes in functional activation. We validated our activation-informed segmentation method in a working memory task setting where ground truth transitions between cognitive states are known. In this validation experiment we found that our method accurately marked known task boundaries, correctly recovered three connectivity states, and displayed a precision and recall profile that compared favorably to a leading sliding window approach. When applying the method to resting state data, we detected five connectivity states that displayed excellent test-retest reliability across four sessions of resting fMRI, exhibited complex transition dynamics, were correlated with multiple behavioral phenotypes, and were essentially unrelated to head motion. Our work expands the methodological toolkit for quantifying and characterizing time-varying connectivity and provides new evidence for the existence of distinct dynamic states during rest.

We assessed the activation-informed segmentation method and sliding window approach head-to-head on a block-design working memory task to test whether these methods detect connectivity state changes where ground truth is known. Laumann et al. performed a test of the opposite issue: They examined a task with extended blocks where connectivity is assumed to be stable and found sliding window methods inappropriately found changing connectivity states where such changes are assumed to be absent (Laumann et al., 2017). In our
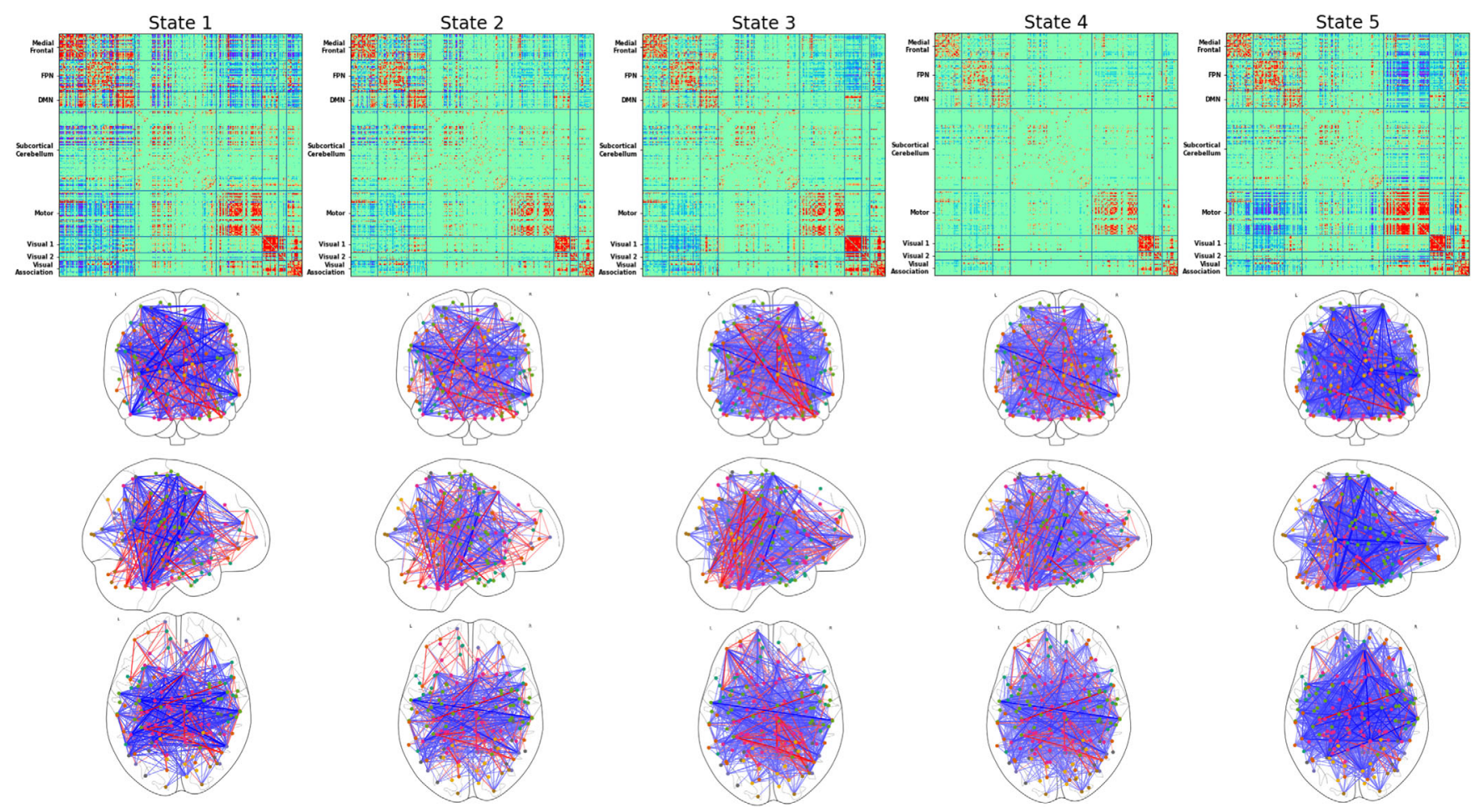

FIGURE 7 Connectivity signatures for each of our five discovered resting FC states. Connectivity signatures are defined by the centroid (i.e., average) of all connectomes belonging to each state cluster. Glass brain views show the top $0.5 \%$ of connections ( 360 edges) for each state 
(a)

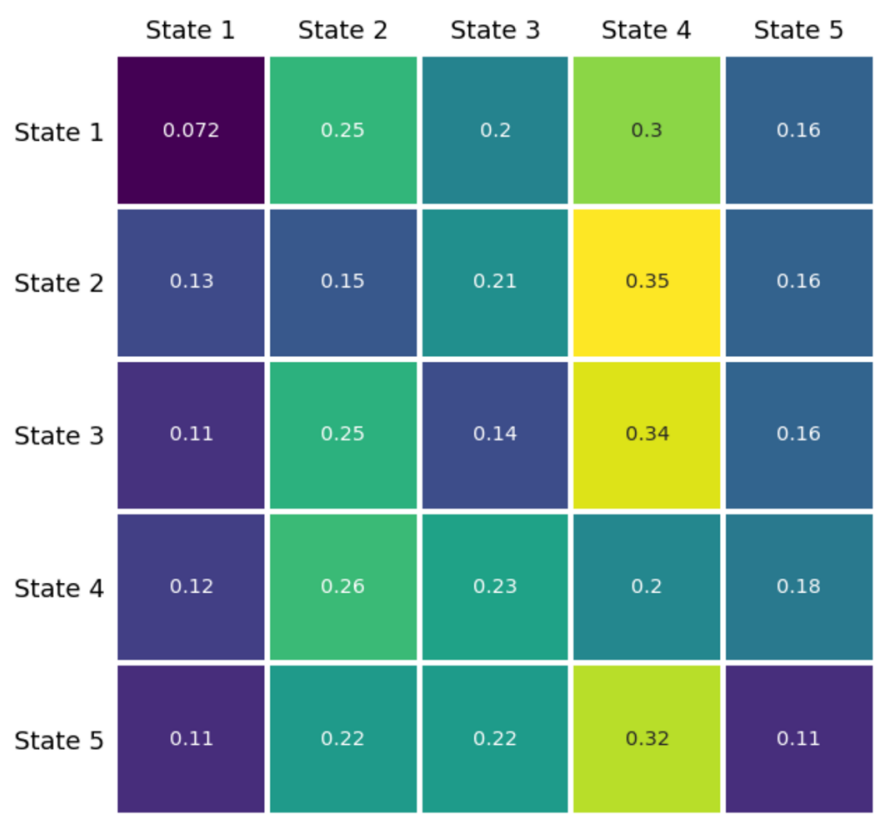

(b)

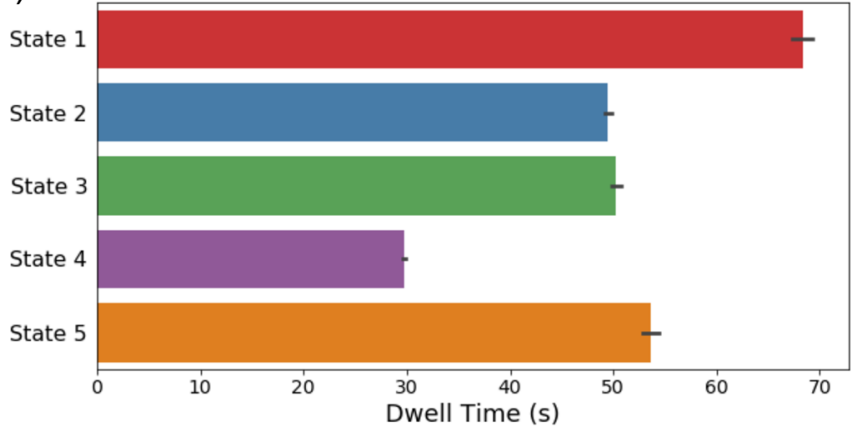

(c)

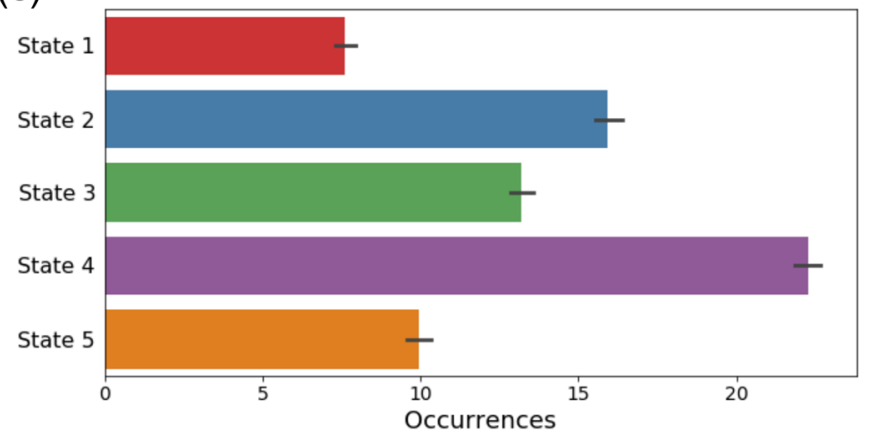

FIG URE 8 Average transition probabilities of moving from State $X$ (along rows) to State $Y$ (along columns) (a), dwell times (b), and number of occurrences (c) across all subjects and resting state fMRI sessions

TAB LE 3 Ordinary least squares regression results for significantly predicted phenotypes (Bonferroni-corrected $\alpha=0.005$ )

\begin{tabular}{|c|c|c|}
\hline Feature & $\beta$ coefficient & $p$-value \\
\hline \multicolumn{3}{|c|}{ Dependent variable $=\mathrm{G} ;$ model F-statistic $=0.000306$} \\
\hline Gender & 0.30 & 0.000 \\
\hline State 1 to State 3 transition probability & -0.144 & 0.041 \\
\hline \multicolumn{3}{|c|}{$\begin{array}{l}\text { Dependent variable }=\text { externalizing behavior; model } \\
\quad F \text {-statistic }=1.56 \mathrm{e}-05\end{array}$} \\
\hline Gender & 2.87 & 0.000 \\
\hline State 3 to State 1 transition probability & -2.57 & 0.008 \\
\hline State 3 to State 2 transition probability & -3.38 & 0.008 \\
\hline Probability of remaining in State 3 & -2.08 & 0.025 \\
\hline State 3 to State 4 transition probability & -3.45 & 0.011 \\
\hline State 3 to State 5 transition probability & -3.24 & 0.004 \\
\hline State 5 to State 1 transition probability & -1.23 & 0.050 \\
\hline State 5 to State 3 transition probability & -2.01 & 0.014 \\
\hline Probability of remaining in State 5 & -1.40 & 0.010 \\
\hline Occurrence of State 5 & 1.20 & 0.039 \\
\hline Age & -0.22 & 0.006 \\
\hline \multicolumn{3}{|c|}{ Dependent variable $=$ agreeableness $;$ model $F$-statistic $=1.94 \mathrm{e}-06$} \\
\hline Gender & -1.91 & 0.000 \\
\hline State 1 to State 5 transition probability & 0.82 & 0.030 \\
\hline \multicolumn{3}{|c|}{ Dependent variable $=$ conscientiousness; model $F$-statistic $=0.00127$} \\
\hline Gender & -1.21 & 0.003 \\
\hline Probability of remaining in State 1 & 0.66 & 0.031 \\
\hline Dwell time State 5 & -0.73 & 0.005 \\
\hline
\end{tabular}

test, the activation-informed segmentation method performed well. We observed an average precision of 0.72 , meaning that $72 \%$ of activation changes detected by our algorithm corresponded to true changes in functional connectivity. Furthermore, the recall of true state transition points by our method averaged 0.66 and reached as high as 0.77 depending on the strength of the functional connectivity changes, indicating that a majority of known connectivity transitions are indeed marked by changes in global functional activation. In contrast, the GIFT sliding window method precludes the calculation of such precision and recall statistics due to the highly overlapping nature of the resultant windows. When considering the accuracy of the final state clustering, our method indeed performed $~ 75 \%$ better than the sliding window method in separating blocks of true task from true rest. As far as we know, this is the first such test of the sliding window method in task data where ground truth is known. The fact that the sliding window has only fair accuracy in finding changes in connectivity state suggests there is room for improvement and reinforces our claim that further methods innovation in the study of time varying $\mathrm{FC}$ would be beneficial.

The activation-informed segmentation method found five states at rest and these states showed excellent test-retest reliability. These states appear to be broadly consistent with those reported in the previous literature in terms of number of states as well as connectivity patterns (Nomi et al., 2017). Furthermore, the mean dwell times are similar in duration. We also found these states are linked to a number of behavioral phenotypes - with the magnitudes of relationships similar to those reported in prior studies (Nomi et al., 2017). Taken together, these results suggest that there is some continuity in our 
results with the results from sliding window approaches. Nonetheless, some key differences remain. First, the states identified here have much higher test-retest reliability. Second, the method to identify them is intuitive, computationally efficient, and appears not to be driven by artifactual causes (e.g., head motion).

A key assumption of our method is that activation changes can serve as a marker of changes in connectivity states. Several lines of evidence support this assumption. First, there is a substantial set of studies (discussed in the Introduction) that document connectivity patterns that arise during distinct task conditions. Importantly, these task conditions are antecedently known to produce distinct distributed activation profiles so that transitions into the relevant task conditions would produce activation shifts. Second, in the present study, we observed GTD peaks during the $\mathrm{N}$-back working memory task when subjects shift task conditions, and we observed distinct connectivity states in the segments flanked by these GTD peaks. Third, if our main assumption were false, that is, if activation shifts fail to mark changes in connectivity states, then we should not have found large activation shifts during rest that are associated with distinct, highly test-retest reliable connectivity states. The fact that we did observe these results from rest provides further support that there is in fact a link between activation shifts and connectivity state changes. Finally, as we noted in the previous paragraph, the states identified have similarities along multiple dimensions with states identified through traditional sliding window methods. If our activation-informed segmentation approach can find connectivity states that are broadly similar to those found by sliding window approaches, this can only be explained if activation changes do indeed serve as a marker of connectivity changes.

In a somewhat unexpected finding, we observed GTD peaks during rest (corresponding to state change points) that were similar in magnitude to those seen during a working memory task. This finding is notable because the $\mathrm{N}$-back working memory task is highly cognitively demanding and produces vigorous activations across a distributed "task-positive network" (Cabeza \& Nyberg, 2000; Cole \& Schneider, 2007; Mazoyer et al., 2001; Niendam et al., 2012). Rest, in contrast, is assumed to be a state of substantially reduced cognitive demands (Andrews-Hanna, Reidler, Huang, \& Buckner, 2010; Buckner, Andrews-Hanna, \& Schacter, 2008; McKiernan, Kaufman, KuceraThompson, \& Binder, 2003). It is thus remarkable that we observed GTD peaks during the resting state on par with those that occur in response to transitions in and out of a cognitively demanding task. The fact that resting GTD peaks are so large provides additional support for our framework, which is based on the idea that easy-to-detect GTD peaks can be leveraged to identify hard-to-detect changes in connectivity states-large GTD peaks are particularly easy to detect. But critically, large GTD peaks during rest should be of independent interest to the field. That is, irrespective of their link to changes in connectivity states (which has been our focus in this study), the fact that there are regular and robust GTD peaks during rest is itself a phenomenon that needs follow up investigation and explanation.

There has been some skepticism in the field about the reality of time varying connectivity. A sizable portion of this debate centers on the sliding window methodology for demonstrating varying connectivity states (Laumann et al., 2017; Lindquist et al., 2014). It is claimed that this method generates artifacts, finds changes where none exist, etc. An important advance of the present study is that it demonstrates time-varying FC during rest without reliance on sliding window methods. Moreover, the associated connectivity states exhibit excellent test-retest reliability. Therefore, we believe that the present study offers some of the strongest evidence to date for the reality of time-varying connectivity at rest. More specifically, we suggest that the state transition points identified by our framework actually represent a lower bound of the "true" dynamic state changes in rest. This is because there is likely only an imperfect relationship between activation shifts and connectivity state changes: the former may be sufficient but not necessary for the latter. Thus, there may be at least some connectivity state changes that are not anteceded by prominent (and thus easy-to-detect) GTD peaks, and our method will fail to detect the presence of such connectivity states. One such example is the transition between Tasks 1 and 2 conditions in the WM task experiment, in which we observed lower recall for the GTD peak detection at these points, indicating these particular connectivity state changes are more subtle and nuanced than transitions from rest to task states and do not result in strong whole brain activation changes. Future work should seek to extend the change point detection scheme developed here to enable identification of these "connectivity-only" transitions. Such a method could be formulated as an extension of existing instantaneous connectivity estimation methodologies (i.e., MTD, ECF, DCC), aimed at identifying significant moment-tomoment changes in multivariate connectivity rather than univariate activation. It is also possible these requirements can be fulfilled through the use of deep learning approaches, specifically recurrent neural network architectures, which are designed to learn complex, nonlinear patterns in multivariate time series data (Li \& Fan, 2018).

This study has several limitations. First, we rely on a key assumption that activation shifts, more specifically those activation shifts that are strong enough to be observed at the whole-brain scale, can serve as a marker for changes in connectivity states. We acknowledge that the relationship is likely imperfect, and our method may underestimate the true number of states. The strength of our method, nonetheless, is simplicity and transparency, enabling the method to yield notably strong evidence for dynamic states at rest. Second, our peak detection scheme is reliant on several empirically tuned parameters as well as an exponentially weighted moving average operation that may be subject to similar criticism as the sliding window Pearson correlation approach. However, it is important to note that the identification of local maxima in a univariate signal (i.e., GTD) is not as sensitive to window size as computation of multivariate cross-correlations-the strongest peaks will survive across a variety of moving average window lengths. Additionally, we note that there are methods available for peak detection that do not rely on moving averages that can be substituted into our pipeline, and future work can explore these approaches. Third, unlike sliding window methods that impose a uniform length on windowed connectivity matrices, the activationinformed segmentation method is sensitive to the duration of states. 
We mitigated this in multiple ways, including Fisher transformation and z-scoring of Pearson correlation-based connectivity matrices, as well as employing a top- $K$ thresholding to control connectome density across both short and long segment lengths. Fourth, the meaning and importance of the dynamic states uncovered by the GTD method is unclear. We showed activation shifts are large (comparable to transitions in and out of a working memory task). We also presented initial data that connectivity states are linked to phenotypes of interest. But additional work is needed to establish what psychological processes are reflected in these dynamic states, and whether quantifying these transient states will yield significant theoretical and practical insights in psychology and neuroscience.

In sum, we introduce here a novel method for identifying dynamic states in $\mathrm{FMRI}$ that generates data-driven segments of stable FC, validate the method in task data where ground truth is known, and demonstrate that the method finds considerable evidence for the presence of dynamic states at rest.

\section{ACKNOWLEDGMENTS}

This study was supported by funding from the University of Michigan Precision Health Investigator Award. Marlena Duda was supported by the National Science Foundation Graduate Research Fellowship Program grant DGE-1256260. The authors thank Parmida Davarmanesh, Aman Taxali, Saige Rutherford and Mike Angstadt for their support and valuable feedback throughout this study.

\section{DATA AVAILABILITY STATEMENT}

Data sharing is not applicable to this article as no new data were created or analyzed in this study.

\section{ORCID}

Marlena Duda (iD https://orcid.org/0000-0003-2369-2225

Chandra Sripada (D) https://orcid.org/0000-0001-9025-6453

\section{REFERENCES}

Allen, E. A., Damaraju, E., Eichele, T., Wu, L., \& Calhoun, V. D. (2018). EEG signatures of dynamic functional network connectivity states. Brain Topography, 31(1), 101-116. https://doi.org/10.1007/s10548-0170546-2

Allen, E. A., Damaraju, E., Plis, S. M., Erhardt, E. B., Eichele, T., \& Calhoun, V. D. (2014). Tracking whole-brain connectivity dynamics in the resting state. Cerebral Cortex (New York, N.Y.: 1991), 24(3), 663676. https://doi.org/10.1093/cercor/bhs352

Andrews-Hanna, J. R., Reidler, J. S., Huang, C., \& Buckner, R. L. (2010). Evidence for the default network's role in spontaneous cognition. Journal of Neurophysiology, 104(1), 322-335. https://doi.org/10.1152/jn. 00830.2009

Baker, A. P., Brookes, M. J., Rezek, I. A., Smith, S. M., Behrens, T., Probert Smith, P. J., \& Woolrich, M. (2014). Fast transient networks in spontaneous human brain activity. eLife, 3, e01867. https://doi.org/10.7554/ elife.01867

Betzel, R. F., Fukushima, M., He, Y., Zuo, X.-N., \& Sporns, O. (2016). Dynamic fluctuations coincide with periods of high and low modularity in resting-state functional brain networks. Neurolmage, 127, 287-297. https://doi.org/10.1016/j.neuroimage.2015.12.001

Biswal, B., Yetkin, F. Z., Haughton, V. M., \& Hyde, J. S. (1995). Functional connectivity in the motor cortex of resting human brain using echo- planar mri. Magnetic Resonance in Medicine, 34(4), 537-541. https:// doi.org/10.1002/mrm.1910340409

Bridgeford, E. W., Wang, S., Yang, Z., Wang, Z., Xu, T., Craddock, C., ... Vogelstein, J. T. (2020). Big data reproducibility: Applications in brain imaging and genomics. bioRxiv, 29. https://www.biorxiv.org/content/ 10.1101/802629v4.abstract

Buckner, R. L., Andrews-Hanna, J. R., \& Schacter, D. L. (2008). The Brain's default network. Annals of the New York Academy of Sciences, 1124(1), 1-38. https://doi.org/10.1196/annals.1440.011

Cabeza, R., \& Nyberg, L. (2000). Imaging cognition II: An empirical review of 275 PET and fMRI studies. Journal of Cognitive Neuroscience, 12(1), $1-47$

Cabral, J., Vidaurre, D., Marques, P., Magalhães, R., Silva Moreira, P., Miguel Soares, J., ... Kringelbach, M. L. (2017). Cognitive performance in healthy older adults relates to spontaneous switching between states of functional connectivity during rest. Scientific Reports, 7, 1-13. https://doi.org/10.1038/s41598-017-05425-7

Cai, B., Zille, P., Stephen, J. M., Wilson, T. W., Calhoun, V. D., \& Wang, Y. P. (2018). Estimation of dynamic sparse connectivity patterns from resting state fMRI. IEEE Transactions on Medical Imaging, 37(5), 1224-1234. https://doi.org/10.1109/TMI.2017.2786553

Calhoun, V. D., Adali, T., Pearlson, G. D., \& Pekar, J. J. (2001). A method for making group inferences from functional MRI data using independent component analysis. Human Brain Mapping, 14(3), 140-151. https://doi.org/10.1002/hbm.1048

Calhoun, V. D., Miller, R., Pearlson, G., \& Adalı, T. (2014). The chronnectome: Time-varying connectivity networks as the next frontier in fMRI data discovery. Neuron, 84(2), 262-274. https://doi.org/ 10.1016/j.neuron.2014.10.015

Chang, C., \& Glover, G. H. (2010). Time-frequency dynamics of restingstate brain connectivity measured with fMRI. Neurolmage, 50(1), 8198. https://doi.org/10.1016/j.neuroimage.2009.12.011

Chang, C., Liu, Z., Chen, M. C., Liu, X., \& Duyn, J. H. (2013). EEG correlates of time-varying BOLD functional connectivity. Neurolmage, 72, 227236. https://doi.org/10.1016/j.neuroimage.2013.01.049

Chen, T., Cai, W., Ryali, S., Supekar, K., \& Menon, V. (2016). Distinct global brain dynamics and spatiotemporal organization of the Salience Network. PLoS Biology, 14(6), e1002469. https://doi.org/10.1371/journal. pbio.1002469

Choe, A. S., Nebel, M. B., Barber, A. D., Cohen, J. R., Xu, Y., Pekar, J. J., ... Lindquist, M. A. (2017). Comparing test-retest reliability of dynamic functional connectivity methods. Neurolmage, 158, 155-175. https:// doi.org/10.1016/j.neuroimage.2017.07.005

Cohen, J. R. (2018). The behavioral and cognitive relevance of time-varying, dynamic changes in functional connectivity. Neurolmage, 180, 515-525. https://doi.org/10.1016/j.neuroimage.2017.09.036

Cole, M. W., \& Schneider, W. (2007). The cognitive control network: Integrated cortical regions with dissociable functions. Neurolmage, 37(1), 343-360. https://doi.org/10.1016/j.neuroimage.2007.03.071

Cordes, D., Zhuang, X., Kaleem, M., Sreenivasan, K., Yang, Z., Mishra, V., ... Cummings, J. L. (2018). Advances in functional magnetic resonance imaging data analysis methods using empirical mode decomposition to investigate temporal changes in early Parkinson's disease. Alzheimer's \& Dementia: Translational Research \& Clinical Interventions, 4, 372-386. https://doi.org/10.1016/j.trci.2018.04.009

Damaraju, E., Allen, E. A., Belger, A., Ford, J. M., McEwen, S., Mathalon, D. H., ... Calhoun, V. D. (2014). Dynamic functional connectivity analysis reveals transient states of dysconnectivity in schizophrenia. Neurolmage: Clinical, 5, 298-308. https://doi.org/10.1016/j.nicl. 2014.07.003

Davison, E. N., Schlesinger, K. J., Bassett, D. S., Lynall, M.-E., Miller, M. B. Grafton, S. T., \& Carlson, J. M. (2015). Brain network adaptability across task states. PLoS Computational Biology, 11(1), e1004029.

Donnat, C., Zitnik, M., Hallac, D., \& Leskovec, J. (2018). Learning structural node embeddings via diffusion wavelets. Paper presented at 
Proceedings of the 24th ACM SIGKDD International conference on Knowledge Discovery \& Data Mining, 1320-1329. https://doi.org/10. $1145 / 3219819.3220025$

Esfahlani, F. Z., Jo, Y., Faskowitz, J., Byrge, L., Kennedy, D. P., Sporns, O., \& Betzel, R. F. (2020). High-amplitude cofluctuations in cortical activity drive functional connectivity. Proceedings of the National Academy of Sciences of the United States of America, 117(45), 28393-28401. https://doi.org/10.1073/pnas.2005531117

Faghiri, A., Iraji, A., Damaraju, E., Belger, A., Ford, J., Mathalon, D., ... Calhoun, V. D. (2020). Weighted average of shared trajectory: A new estimator for dynamic functional connectivity efficiently estimates both rapid and slow changes over time. Journal of Neuroscience Methods, 334, 108600. https://doi.org/10.1016/j.jneumeth.2020. 108600

Finn, E. S., Shen, X., Scheinost, D., Rosenberg, M. D., Huang, J., Chun, M. M., ... Constable, R. T. (2015). Functional connectome fingerprinting: Identifying individuals using patterns of brain connectivity. Nature Neuroscience, 18(11), 1664-1671. https://doi.org/10.1038/nn. 4135

Fisher, R. A. (1915). Frequency distribution of the values of the correlation coefficient in samples from an indefinitely large population. Biometrika, 10(4), 507-521. https://doi.org/10.2307/2331838

Friston, K. J. (2003). Statistical parametric mapping. In R. Kötter (Ed.), Neuroscience databases: A practical guide (pp. 237-250). USA: Springer. https://doi.org/10.1007/978-1-4615-1079-6_16

Glasser, M. F., Sotiropoulos, S. N., Wilson, J. A., Coalson, T. S., Fischl, B., Andersson, J. L., ... WU-Minn HCP Consortium. (2013). The minimal preprocessing pipelines for the human connectome project. Neurolmage, 80, 105-124. https://doi.org/10.1016/j.neuroimage.2013.04.127

Gonzalez-Castillo, J., Hoy, C. W., Handwerker, D. A., Robinson, M. E., Buchanan, L. C., Saad, Z. S., \& Bandettini, P. A. (2015). Tracking ongoing cognition in individuals using brief, whole-brain functional connectivity patterns. Proceedings of the National Academy of Sciences of the United States of America, 112(28), 8762-8767.

Greicius, M. D., Krasnow, B., Reiss, A. L., \& Menon, V. (2003). Functional connectivity in the resting brain: A network analysis of the default mode hypothesis. Proceedings of the National Academy of Sciences of the United States of America, 100(1), 253-258. https://doi.org/10. 1073/pnas.0135058100

Hafner, C. M., \& Reznikova, O. (2012). On the estimation of dynamic conditional correlation models. Computational Statistics \& Data Analysis, 56(11), 3533-3545. https://doi.org/10.1016/j.csda.2010.09.022

Hindriks, R., Adhikari, M. H., Murayama, Y., Ganzetti, M., Mantini, D., Logothetis, N. K., \& Deco, G. (2016). Can sliding-window correlations reveal dynamic functional connectivity in resting-state fMRI? Neurolmage, 127, 242-256. https://doi.org/10.1016/j.neuroimage. 2015.11.055

Hull, J. V., Dokovna, L. B., Jacokes, Z. J., Torgerson, C. M., Irimia, A., \& Van Horn, J. D. (2017). Resting-state functional connectivity in autism Spectrum disorders: A review. Frontiers in Psychiatry, 7, 205. https:// doi.org/10.3389/fpsyt.2016.00205

Hutchison, R. M., \& Morton, J. B. (2015). Tracking the Brain's functional coupling dynamics over development. Journal of Neuroscience, 35(17), 6849-6859. https://doi.org/10.1523/JNEUROSCI.4638-14.2015

Hutchison, R. M., Womelsdorf, T., Allen, E. A., Bandettini, P. A., Calhoun, V. D., Corbetta, M., ... Chang, C. (2013). Dynamic functional connectivity: Promise, issues, and interpretations. Neurolmage, 80, 360-378. https://doi.org/10.1016/j.neuroimage.2013.05.079

Jones, D. T., Vemuri, P., Murphy, M. C., Gunter, J. L., Senjem, M. L., Machulda, M. M., ... Jr, C. R. J. (2012). Non-stationarity in the "resting Brain's" modular architecture. PLoS One, 7(6), e39731. https://doi.org/ 10.1371/journal.pone.0039731

Laumann, T. O., Snyder, A. Z., Mitra, A., Gordon, E. M., Gratton, C., Adeyemo, B., ... Petersen, S. E. (2017). On the stability of BOLD fMRI correlations. Cerebral Cortex (New York, NY), 27(10), 4719-4732. https://doi.org/10.1093/cercor/bhw265

Li, H., \& Fan, Y. (2018). Identification of temporal transition of functional states using recurrent neural networks from functional MRI. ArXiv:1809.05560 [Cs, q-Bio]. http://arxiv.org/abs/1809. 05560

Lindquist, M. A., Xu, Y., Nebel, M. B., \& Caffo, B. S. (2014). Evaluating dynamic bivariate correlations in resting-state fMRI: A comparison study and a new approach. Neurolmage, 101, 531-546. https://doi. org/10.1016/j.neuroimage.2014.06.052

Liu, X., \& Duyn, J. H. (2013). Time-varying functional network information extracted from brief instances of spontaneous brain activity. Proceedings of the National Academy of Sciences of the United States of America, 110(11), 4392-4397. https://doi.org/10.1073/pnas. 1216856110

Lurie, D. J., Kessler, D., Bassett, D. S., Betzel, R. F., Breakspear, M., Kheilholz, S., ... Calhoun, V. D. (2019). Questions and controversies in the study of time-varying functional connectivity in resting fMRI. Network Neuroscience, 4(1), 30-69. https://doi.org/10.1162/netn_a_ 00116

Lynall, M.-E., Bassett, D. S., Kerwin, R., McKenna, P. J., Kitzbichler, M., Muller, U., \& Bullmore, E. (2010). Functional Connectivity and Brain Networks in Schizophrenia. Journal of Neuroscience, 30(28), 9477-9487. https://doi.org/10.1523/JNEUROSCI.0333-10.2010

Mao, N., Zheng, H., Long, Z., Yao, L., \& Wu, X. (2017). Gender differences in dynamic functional connectivity based on resting-state fMRI. Annual International Conference of the IEEE Engineering in Medicine and Biology Society. IEEE Engineering in Medicine and Biology Society, 2940-2943. https://doi.org/10.1109/EMBC.2017.8037473

Marusak, H. A., Calhoun, V. D., Brown, S., Crespo, L. M., Sala-Hamrick, K., Gotlib, I. H., \& Thomason, M. E. (2016). Dynamic functional connectivity of neurocognitive networks in children. Human Brain Mapping, 38(1), 97-108. https://doi.org/10.1002/hbm.23346

Marusak, H. A., Elrahal, F., Peters, C. A., Kundu, P., Lombardo, M. V., Calhoun, V. D., ... Rabinak, C. A. (2018). Mindfulness and dynamic functional neural connectivity in children and adolescents. Behavioural Brain Research, 336, 211-218. https://doi.org/10.1016/j.bbr.2017. 09.010

Mazoyer, B., Zago, L., Mellet, E., Bricogne, S., Etard, O., Houdé, O., ... Tzourio-Mazoyer, N. (2001). Cortical networks for working memory and executive functions sustain the conscious resting state in man. Brain Research Bulletin, 54(3), 287-298.

McKiernan, K. A., Kaufman, J. N., Kucera-Thompson, J., \& Binder, J. R. (2003). A parametric manipulation of factors affecting task-induced deactivation in functional neuroimaging. Journal of Cognitive Neuroscience, 15, 394-408.

Mokhtari, F., Akhlaghi, M. I., Simpson, S. L., Wu, G., \& Laurienti, P. J. (2019). Sliding window correlation analysis: Modulating window shape for dynamic brain connectivity in resting state. Neurolmage, 189, 655666. https://doi.org/10.1016/j.neuroimage.2019.02.001

Niendam, T. A., Laird, A. R., Ray, K. L., Dean, Y. M., Glahn, D. C., \& Carter, C. S. (2012). Meta-analytic evidence for a superordinate cognitive control network subserving diverse executive functions. Cognitive, Affective, \& Behavioral Neuroscience, 12(2), 241-268. https://doi.org/ 10.3758/s13415-011-0083-5

Nomi, J. S., Vij, S. G., Dajani, D. R., Steimke, R., Damaraju, E., Rachakonda, S., ... Uddin, L. Q. (2017). Chronnectomic patterns and neural flexibility underlie executive function. Neurolmage, 147, 861-871. https://doi.org/10.1016/ j.neuroimage.2016.10.026

Pedersen, M., Omidvarnia, A., Zalesky, A., \& Jackson, G. D. (2018). On the relationship between instantaneous phase synchrony and correlationbased sliding windows for time-resolved fMRI connectivity analysis. Neurolmage, 181, 85-94. https://doi.org/10.1016/j.neuroimage.2018. 06.020 
Power, J. D., Barnes, K. A., Snyder, A. Z., Schlaggar, B. L., \& Petersen, S. E. (2012). Spurious but systematic correlations in functional connectivity MRI networks arise from subject motion. Neurolmage, 59(3), 2142-2154. https://doi.org/10.1016/j.neuroimage.2011.10.018

Power, J. D., Schlaggar, B. L., \& Petersen, S. E. (2015). Recent progress and outstanding issues in motion correction in resting state $\mathrm{fMRI}$. Neurolmage, 105, 536-551. https://doi.org/10.1016/j.neuroimage. 2014.10.044

Preti, M. G., Bolton, T. A., \& Van De Ville, D. (2017). The dynamic functional connectome: State-of-the-art and perspectives. Neurolmage, 160, 41-54. https://doi.org/10.1016/j.neuroimage.2016.12.061

Quinn, A. J., Vidaurre, D., Abeysuriya, R., Becker, R., Nobre, A. C., \& Woolrich, M. W. (2018). Task-evoked dynamic network analysis through hidden Markov modeling. Frontiers in Neuroscience, 12, 603. https://doi.org/10.3389/fnins.2018.00603

Rashid, B., Damaraju, E., Pearlson, G. D., \& Calhoun, V. D. (2014). Dynamic connectivity states estimated from resting fMRI identify differences among schizophrenia, bipolar disorder, and healthy control subjects. Frontiers in Human Neuroscience, 8, 897. https://doi.org/10.3389/ fnhum.2014.00897

Rossi, R. A., Jin, D., Kim, S., Ahmed, N. K., Koutra, D., \& Lee, J. B. (2020). On proximity and structural role-based embeddings in networks: Misconceptions, techniques, and applications. ACM Transactions on Knowledge Discovery from Data, 14(5), 1-37. https://doi.org/10.1145/ 3397191

Salimi-Khorshidi, G., Douaud, G., Beckmann, C. F., Glasser, M. F., Griffanti, L., \& Smith, S. M. (2014). Automatic denoising of functional MRI data: Combining independent component analysis and hierarchical fusion of classifiers. Neurolmage, 90, 449-468. https://doi.org/10. 1016/j.neuroimage.2013.11.046

Satterthwaite, T. D., Wolf, D. H., Loughead, J., Ruparel, K., Elliott, M. A., Hakonarson, H., ... Gur, R. E. (2012). Impact of in-scanner head motion on multiple measures of functional connectivity: Relevance for studies of neurodevelopment in youth. Neurolmage, 60(1), 623-632. https:// doi.org/10.1016/j.neuroimage.2011.12.063

Savva, A. D., Mitsis, G. D., \& Matsopoulos, G. K. (2019). Assessment of dynamic functional connectivity in resting-state fMRI using the sliding window technique. Brain and Behavior, 9(4), e01255. https://doi.org/ 10.1002/brb3.1255

Schönbrodt, F. D., \& Perugini, M. (2013). At what sample size do correlations stabilize? Journal of Research in Personality, 47(5), 609-612. https://doi.org/10.1016/j.jrp.2013.05.009

Shakil, S., Billings, J. C., Keilholz, S. D., \& Lee, C.-H. (2018). Parametric dependencies of sliding window correlation. IEEE Transactions on Biomedical Engineering, 65(2), 254-263. https://doi.org/10.1109/TBME. 2017.2762763

Shakil, S., Keilholz, S. D., \& Lee, C.-H. (2015). On frequency dependencies of sliding window correlation. Paper presente at: 2015 IEEE International Conference on Bioinformatics and Biomedicine (BIBM), 363368. https://doi.org/10.1109/BIBM.2015.7359708

Shakil, S., Lee, C.-H., \& Keilholz, S. D. (2016). Evaluation of sliding window correlation performance for characterizing dynamic functional connectivity and brain states. Neurolmage, 133, 111-128. https://doi.org/10. 1016/j.neuroimage.2016.02.074

Shine, J. M., Koyejo, O., Bell, P. T., Gorgolewski, K. J., Gilat, M., \& Poldrack, R. A. (2015). Estimation of dynamic functional connectivity using multiplication of temporal derivatives. Neurolmage, 122, 399407. https://doi.org/10.1016/j.neuroimage.2015.07.064

Shine, J. M., \& Poldrack, R. A. (2018). Principles of dynamic network reconfiguration across diverse brain states. Neurolmage, 180, 396-405.

Shou, H., Eloyan, A., Lee, S., Zipunnikov, V., Crainiceanu, A. N., Nebel, N. B., ... Crainiceanu, C. M. (2013). Quantifying the reliability of image replication studies: The image intraclass correlation coefficient (I2C2). Cognitive, Affective, \& Behavioral Neuroscience, 13(4), 714-724. https://doi.org/10. 3758/s13415-013-0196-0

Siegel, J. S., Mitra, A., Laumann, T. O., Seitzman, B. A., Raichle, M., Corbetta, M., \& Snyder, A. Z. (2017). Data quality influences observed links between functional connectivity and behavior. Cerebral Cortex (New York, N.Y.: 1991), 27(9), 4492-4502. https://doi.org/10.1093/ cercor/bhw253

Smith, D. M., Zhao, Y., Keilholz, S. D., \& Schumacher, E. H. (2018). Investigating the intersession reliability of dynamic brain-state properties. Brain Connectivity, 8(5), 255-267. https://doi.org/10.1089/brain. 2017.0571

Spielberg, J. M., Miller, G. A., Heller, W., \& Banich, M. T. (2015). Flexible brain network reconfiguration supporting inhibitory control. Proceedings of the National Academy of Sciences of the United States of America, 112(32), 10020-10025.

Sripada, C., Angstadt, M., Kessler, D., Phan, K. L., Liberzon, I., Evans, G. W., ... Swain, J. E. (2014). Volitional regulation of emotions produces distributed alterations in connectivity between visual, attention control, and default networks. Neurolmage, 89, 110-121. https://doi.org/10. 1016/j.neuroimage.2013.11.006

Sripada, C., Angstadt, M., Rutherford, S., Kessler, D., Kim, Y., Yee, M., \& Levina, E. (2019). Basic units of inter-individual variation in resting state connectomes. Scientific Reports, 9(1), 1900. https://doi.org/10. 1038/s41598-018-38406-5

Sripada, C., Angstadt, M., Rutherford, S., Taxali, A., \& Shedden, K. (2020). Toward a "treadmill test" for cognition: Improved prediction of general cognitive ability from the task activated brain. Human Brain Mapping, 41(12).

Tagliazucchi, E., von Wegner, F., Morzelewski, A., Brodbeck, V., \& Laufs, H. (2012). Dynamic BOLD functional connectivity in humans and its electrophysiological correlates. Frontiers in Human Neuroscience, 6, 339. https://doi.org/10.3389/fnhum.2012.00339

Telesford, Q. K., Lynall, M.-E., Vettel, J., Miller, M. B., Grafton, S. T., \& Bassett, D. S. (2016). Detection of functional brain network reconfiguration during task-driven cognitive states. Neurolmage, 142, 198-210.

Thirion, B., Pinel, P., Mériaux, S., Roche, A., Dehaene, S., \& Poline, J.-B. (2007). Analysis of a large fMRI cohort: Statistical and methodological issues for group analyses. Neurolmage, 35(1), 105-120. https://doi. org/10.1016/j.neuroimage.2006.11.054

Turner, B. O., Paul, E. J., Miller, M. B., \& Barbey, A. K. (2018). Small sample sizes reduce the replicability of task-based $\mathrm{fMRI}$ studies. Communications Biology, 1, 1-10. https://doi.org/10.1038/s42003-018-0073-z

van Dijk, K. R. A., Sabuncu, M. R., \& Buckner, R. L. (2012). The influence of head motion on intrinsic functional connectivity MRI. Neurolmage, 59(1), 431-438. https://doi.org/10.1016/j.neuroimage.2011.07.044

Van Essen, D. C., Smith, S. M., Barch, D. M., Behrens, T. E. J., Yacoub, E., \& Ugurbil, K. (2013). The WU-Minn human connectome project: An overview. Neurolmage, 80, 62-79. https://doi.org/10.1016/j. neuroimage.2013.05.041

Vidaurre, D., Smith, S. M., \& Woolrich, M. W. (2017). Brain network dynamics are hierarchically organized in time. Proceedings of the National Academy of Sciences of the United States of America, 114(48), 12827-12832. https://doi.org/10.1073/pnas.1705120114

Wong, C. W., Olafsson, V., Tal, O., \& Liu, T. T. (2013). The amplitude of the resting-state $\mathrm{fMRI}$ global signal is related to EEG vigilance measures. Neurolmage, 83, 983-990. https://doi.org/10.1016/j.neuroimage. 2013.07.057

Zalesky, A., Fornito, A., Cocchi, L., Gollo, L. L., \& Breakspear, M. (2014). Time-resolved resting-state brain networks. Proceedings of the National Academy of Sciences, 111(28), 10341-10346. https://doi.org/10. 1073/pnas.1400181111 
Zhang, G., Cai, B., Zhang, A., Stephen, J. M., Wilson, T. W., Calhoun, V. D., \& Wang, Y.-P. (2020). Estimating dynamic functional brain connectivity with a sparse hidden Markov model. IEEE Transactions on Medical Imaging, 39(2), 488-498. https://doi.org/10.1109/ TMI.2019.2929959

\section{SUPPORTING INFORMATION}

Additional supporting information may be found in the online version of the article at the publisher's website.
How to cite this article: Duda, M., Koutra, D., \& Sripada, C. (2021). Validating dynamicity in resting state fMRI with activation-informed temporal segmentation. Human Brain Mapping, 42(17), 5718-5735. https://doi.org/10.1002/hbm. 25649 